\title{
FROM THE DISCRETE TO THE CONTINUOUS COAGULATION-FRAGMENTATION EQUATIONS
}

\author{
Philippe Laurençot ${ }^{1}$ and Stéphane Mischler ${ }^{2}$
}

\begin{abstract}
The connection between the discrete and the continuous coagulation-fragmentation models is investigated. A weak stability principle relying on $a$ priori estimates and weak compactness in $L^{1}$ is developed for the continuous model. We approximate the continuous model by a sequence of discrete models and, writing the discrete models as modified continuous ones, we prove the convergence of the latter towards the former with the help of the above mentioned stability principle. Another application of this stability principle is the convergence of an explicit time and size discretisation of the continuous coagulation-fragmentation model.
\end{abstract}

\section{Introduction}

Coagulation and fragmentation processes arise in the dynamics of cluster growth and describe the mechanisms by which clusters can coalesce to form larger clusters or break apart into smaller pieces. In the simplest coagulation-fragmentation models the clusters are usually assumed to be fully identified by their size (or volume or number of particles) which might be either a positive real number (continuous models) or a positive integer (discrete models) depending on the physical context. Though the relationship between the discrete and the continuous models has been considered by some authors, see the survey paper [11, p. 127] and $[1,5,6,36]$, their analysis is either performed at a formal level $[1,11]$ or their approach is restricted either to a particular fragmentation model (scaling technique [36]) or to the coagulation model (via measure-valued solutions) in [5]. The aim of this paper is to provide a rigorous setting for the formal analysis performed in $[1,11]$ under general assumptions on the coagulation and fragmentation coefficients. A related approach motivated by the study of a numerical scheme is developed in [6] for the coagulation equation with very restrictive assumptions on the coagulation coefficients.

\footnotetext{
${ }^{1}$ Mathématiques pour l'Industrie et la Physique, CNRS UMR 5640, Université Paul Sabatier Toulouse 3, 118 route de Narbonne, F-31062 Toulouse cedex 4, France

E-mail : laurencot@mip.ups-tlse.fr

${ }^{2}$ Laboratoire de Mathématiques Appliquées, Université de Versailles - Saint Quentin, 45 avenue des Etats-Unis, F-78035 Versailles, France and DMA, CNRS UMR 8553, Ecole Normale Supérieure, 45 rue d'Ulm, F-75230 Paris cedex 05, France

E-mail : stephane.mischler@ens.fr
} 
The coagulation-fragmentation models we consider in this paper describe the time evolution of the cluster size distribution as the system of clusters undergoes binary coagulation and binary fragmentation events. More precisely, denoting by $C_{z}$ the clusters of size $z$ with $z=y \in \mathbb{R}_{+}=(0,+\infty)$ or $z=i \in \mathbb{N} \backslash\{0\}$, the basic reactions taken into account herein are

$$
C_{z}+C_{z^{\prime}} \stackrel{a\left(z, z^{\prime}\right)}{\longrightarrow} C_{z+z^{\prime}} \quad \text { (binary coagulation), }
$$

and

$$
C_{z} \stackrel{b\left(z-z^{\prime}, z^{\prime}\right)}{\longrightarrow} C_{z-z^{\prime}}+C_{z^{\prime}} \quad \text { (binary fragmentation), }
$$

where $a$ and $b$ denote the coagulation and fragmentation rates, respectively, and are assumed to depend only on the sizes of the clusters involved in these reactions.

In the continuous setting, denoting by $f(t, y)$ the size distribution function at time $t$, the continuous coagulation-fragmentation equation (hereafter refered to as the $\mathrm{CCF}$ equation) reads

$$
\begin{aligned}
\frac{\partial f}{\partial t} & =Q(f), \quad(t, y) \in(0,+\infty) \times \mathbb{R}_{+}, \\
f(0, y) & =f^{i n}(y), \quad y \in \mathbb{R}_{+} .
\end{aligned}
$$

Here the coagulation-fragmentation reaction term $Q(f)$ is given by

$$
Q(f)=Q_{1}(f)-Q_{2}(f)-Q_{3}(f)+Q_{4}(f),
$$

with

$$
\begin{aligned}
& Q_{1}(f)(y)=\frac{1}{2} \int_{0}^{y} a\left(y^{\prime}, y-y^{\prime}\right) f\left(y^{\prime}\right) f\left(y-y^{\prime}\right) d y^{\prime} \\
& Q_{2}(f)(y)=\frac{1}{2} \int_{0}^{y} b\left(y^{\prime}, y-y^{\prime}\right) d y^{\prime} f(y), \\
& Q_{3}(f)(y)=L(f)(y) f(y) \\
& \text { with } \quad L(f)(y):=\int_{0}^{\infty} a\left(y, y^{\prime}\right) f\left(y^{\prime}\right) d y^{\prime}, \\
& Q_{4}(f)(y)=\int_{0}^{\infty} b\left(y, y^{\prime}\right) f\left(y+y^{\prime}\right) d y^{\prime} .
\end{aligned}
$$

The meaning of the different contributions to the reaction term $Q(f)$ are the following : $Q_{1}(f)$ accounts for the formation of clusters $C_{y}$ by coalescence of smaller clusters and $Q_{2}(f)$ for the breakage of clusters $C_{y}$ into two smaller pieces. The term $Q_{3}(f)$ describes the depletion of clusters $C_{y}$ by coagulation with other clusters, while $Q_{4}(f)$ represents the gain of clusters $C_{y}$ as a result of the fragmentation of larger clusters.

In the discrete setting, the size distribution function $c_{i}(t)$ of clusters of size $i \in \mathbb{N} \backslash\{0\}$ (or $i$-clusters) at time $t \geq 0$ obeys the following system of discrete coagulation-fragmentation equations (hereafter refered to as the DCF equations)

$$
\frac{d c_{i}}{d t}=Q_{i}(c) \text { in }(0,+\infty), \quad c_{i}(0)=c_{i}^{i n},
$$


for $i \geq 1$, where

$$
Q_{i}(c)=Q_{1, i}(c)-Q_{2, i}(c)-Q_{3, i}(c)+Q_{4, i}(c), \quad i \geq 1,
$$

with

$$
\begin{aligned}
& Q_{1, i}(c)=\frac{1}{2} \sum_{j=1}^{i-1} a_{i-j, j} c_{i-j} c_{j}, \quad Q_{2, i}(c)=\frac{1}{2} \sum_{j=1}^{i-1} b_{i-j, j} c_{i}, \\
& Q_{3, i}(c)=L_{i}(c) c_{i} \text { with } L_{i}(c)=\sum_{j=1}^{\infty} a_{i, j} c_{j}, \quad Q_{4, i}(c)=\sum_{j=1}^{\infty} b_{i, j} c_{i+j} .
\end{aligned}
$$

The reaction terms $Q_{k, i}(c)$ have a similar meaning to that of $Q_{k}(f), k \in\{1, \ldots, 4\}$. In fact the DCF equations (1.4)-(1.5) have been originally derived by Smoluchowski $[30,31]$ without the fragmentation term $\left(b_{i, j}=0\right)$ to describe the coalescence of colloids moving according to a Brownian movement. It had subsequently been extended to the continuous setting by Müller [26] (see also [11] for a more detailed historical viewpoint). Since then, both DCF and CCF equations have been used in a wide variety of physical and biological situations, including aerosol physics (rain drops formation,...), polymer chemistry, astrophysics (formation of the stars and the planets), hematology or population dynamics (animal grouping). The choice of the size range $\left(\mathbb{N} \backslash\{0\}\right.$ or $\left.\mathbb{R}_{+}\right)$is then peculiar to the scale of the phenomenon to be described, but also depends on the desired level of description (so that both equations may be used to model the same phenomenon but at different scales). It thus seems to be relevant to investigate precisely the connection between the two approaches.

We now present the main idea upon which our approach is built. We actually adapt a method introduced for the Boltzmann equation in [25] (see also [28] and the references therein for further developments) and show that solutions to the DCF equations satisfy a "modified" CCF equation. More precisely, let us start with a solution $c=\left(c_{i}\right)$ to the DCF equations (1.4)-(1.5). For any sequence $\left(\varphi_{i}\right)$ of real numbers (decaying sufficiently rapidly for large values of $i \in \mathbb{N}$ ) we have

$$
\frac{d}{d t} \sum_{i=1}^{\infty} c_{i} \varphi_{i}=\frac{1}{2} \sum_{i, j=1}^{\infty}\left(a_{i j} c_{i} c_{j}-b_{i j} c_{i+j}\right)\left(\varphi_{i+j}-\varphi_{i}-\varphi_{j}\right) .
$$

Note that (1.6) is a weak formulation of the DCF equations (1.4)-(1.5) and may be taken as the definition of a (weak) solution to the DCF equations (1.4)-(1.5).

In order to interpret (1.6) as the weak formulation of a "modified" CCF equation we introduce some notations. We fix $\varepsilon \in(0,1)$ and define

$$
f_{\varepsilon}(t, y)=\sum_{i=1}^{\infty} c_{i}(t) \chi_{i}^{\varepsilon}(y)
$$

and

$$
a_{\varepsilon}\left(y, y^{\prime}\right)=\sum_{i, j=1}^{\infty} \frac{a_{i, j}}{\varepsilon} \chi_{i}^{\varepsilon}(y) \chi_{j}^{\varepsilon}\left(y^{\prime}\right), \quad b_{\varepsilon}\left(y, y^{\prime}\right)=\sum_{i, j=1}^{\infty} \frac{b_{i, j}}{\varepsilon} \chi_{i}^{\varepsilon}(y) \chi_{j}^{\varepsilon}\left(y^{\prime}\right),
$$


for $\left(t, y, y^{\prime}\right) \in \mathbb{R}_{+}^{3}$ where we have set

$$
\Lambda_{i}^{\varepsilon}=[(i-1 / 2) \varepsilon,(i+1 / 2) \varepsilon), \quad \chi_{i}^{\varepsilon}=\mathbf{1}_{\Lambda_{i}^{\varepsilon}}, \quad i \geq 1 .
$$

Next, for $\varphi \in \mathcal{D}\left(\mathbb{R}_{+}\right)$, we define the sequence $\left(\varphi_{\varepsilon}\right)$ of functions by

$$
\varphi_{\varepsilon}(y)=\sum_{i=1}^{\infty} \varphi_{i}^{\varepsilon} \chi_{i}^{\varepsilon}(y), \quad \varphi_{i}^{\varepsilon}=\frac{1}{\varepsilon} \int_{\Lambda_{i}^{\varepsilon}} \varphi(y) d y .
$$

Finally, for any $\varepsilon$-step function $g$, that is, $g$ is a measurable function from $\mathbb{R}_{+}$to $\mathbb{R}$ such that

$$
g(y)=\sum_{i=1}^{\infty} g_{i} \chi_{i}^{\varepsilon}(y), \quad g_{i} \in \mathbb{R},
$$

we define

$$
T_{\varepsilon}(g)\left(y, y^{\prime}\right):=\sum_{i, j=1}^{\infty} g_{i+j} \chi_{i}^{\varepsilon}(y) \chi_{j}^{\varepsilon}\left(y^{\prime}\right), \quad\left(y, y^{\prime}\right) \in \mathbb{R}_{+}^{2} .
$$

Let us emphasize here that $T_{\varepsilon}(g)\left(y, y^{\prime}\right)$ must be seen as an approximation of $g\left(y+y^{\prime}\right)$ (see Lemma 4.1 below).

With these notations we are in a position to write an alternative formulation of (1.6) in terms of the new functions $f_{\varepsilon}, a_{\varepsilon}, b_{\varepsilon}$ and $\varphi_{\varepsilon}$ which reads

(1.13) $\frac{d}{d t} \int_{0}^{\infty} f_{\varepsilon} \varphi_{\varepsilon} d y=\frac{1}{2} \int_{0}^{\infty} \int_{0}^{\infty}\left(a_{\varepsilon} f_{\varepsilon} f_{\varepsilon}^{\prime}-b_{\varepsilon} T_{\varepsilon}\left(f_{\varepsilon}\right)\right)\left(T_{\varepsilon}\left(\varphi_{\varepsilon}\right)-\varphi_{\varepsilon}-\varphi_{\varepsilon}^{\prime}\right) d y d y^{\prime}$.

Here and below we use the following notation :

$$
g=g(y), \quad g^{\prime}=g\left(y^{\prime}\right) \text { and } g^{\prime \prime}=g\left(y+y^{\prime}\right) .
$$

We first prove that, under some growth conditions on $a_{i j}$ and $b_{i j},\left(f_{\varepsilon}\right)$ lies in a weakly compact subset of $L^{1}\left((0, T) \times \mathbb{R}_{+}\right)$for each $T>0$ : consequently there exist $f \in L_{\mathrm{loc}}^{1}\left([0, \infty) \times \mathbb{R}_{+}\right)$and a subsequence of $\left(f_{\varepsilon}\right)$ (not relabeled) such that

$$
f_{\varepsilon} \rightarrow f \quad \text { weakly in } L^{1}\left((0, T) \times \mathbb{R}_{+}\right) \quad \text { for each } T \in \mathbb{R}_{+} .
$$

Now, if there are some functions $a$ and $b$ such that

$$
a_{\varepsilon} \rightarrow a, \quad b_{\varepsilon} \rightarrow b \quad \text { a.e. and weakly in } L_{\mathrm{loc}}^{\infty}\left(\mathbb{R}_{+}^{2}\right),
$$

we are able to pass to the limit in (1.13) and obtain that $f$ satisfies

$$
\frac{d}{d t} \int_{0}^{\infty} f \varphi d y=\frac{1}{2} \int_{0}^{\infty} \int_{0}^{\infty}\left(a f f^{\prime}-b f^{\prime \prime}\right)\left(\varphi^{\prime \prime}-\varphi-\varphi^{\prime}\right) d y d y^{\prime}
$$

in $\mathcal{D}^{\prime}([0,+\infty))$ for any $\varphi \in \mathcal{D}\left(\mathbb{R}_{+}\right)$. In other words, $f$ is a (weak) solution to the CCF equation (1.1)-(1.3). To be more precise we actually proceed as follows : given coagulation and fragmentation rates $a$ and $b$ we construct a family of discrete 
kinetic coefficients $\left(a_{i, j}^{\varepsilon}\right)$ and $\left(b_{i, j}^{\varepsilon}\right)$ in such a way that (1.14) and (1.15) hold and thus establish the expected connection between the DCF and CCF equations. In fact, as for the Boltzmann equation, the underlying idea is somehow a stability principle : the weak $L^{1}$-compactness of sequences of solutions to the CCF equation is also enjoyed by sequences of solutions to suitable perturbations of the CCF equation. In particular, the DCF equations (1.4)-(1.5) are such a perturbation. Furthermore, it turns out that an Euler explicit time discretization of the DCF equations also fits into the framework developed in this paper and the convergence of this scheme towards a solution to the CCF equation is studied below. Let us mention at this point that, though several numerical simulations of the CCF equation have been performed with various deterministic or stochastic numerical methods (see, e.g., [3, $10,14,18,24,27,29]$ and the references therein), convergence proofs have only been supplied recently and we refer the reader to, e.g., $[8,13,14,17,29]$ for the analysis of stochastic algorithms. As for deterministic numerical schemes, the only result we are aware of concerns a time explicit Euler scheme for the discrete coagulation equations $\left(b_{i, j} \equiv 0\right)$ which is shown to converge for bounded coefficients $\left(a_{i, j}\right)[29]$. Within our approach, such a restriction is not necessary and the convergence of the time explicit scheme presented below is valid under fairly general assumptions on the kinetic coefficients $a$ and $b$.

Let us now briefly outline the contents of the paper : the construction of the sequence of DCF equations approximating the CCF equation is described in the next section where our convergence results are stated as well. Let us emphasize here that, besides the connection between the DCF and the CCF equations, we also establish new existence results for the $\mathrm{CCF}$ equation as a by-product. The a priori estimates guaranteeing the weak compactness of $\left(f_{\varepsilon}\right)$ in $L^{1}$ are gathered in Section 3 while the passage to the limit is performed in Section 4. As already mentioned, our approach is quite general and we discuss several extensions in the next sections. In Section 5 we show how a similar approach allows us to obtain the convergence of a time explicit Euler scheme. In the remaining sections we outline how one can handle other classes of coagulation and fragmentation rates (Section 6) and how our method may be applied to the coagulation-fragmentation equations with diffusion (Section 7). We finally discuss in the Appendix the equivalence between two seemingly different notions of solutions to the CCF equation.

\section{Main results}

Throughout the paper we make the following symmetry and growth assumptions

$$
\begin{aligned}
& a\left(y, y^{\prime}\right)=a\left(y^{\prime}, y\right) \text { and } b\left(y, y^{\prime}\right)=b\left(y^{\prime}, y\right), \quad\left(y, y^{\prime}\right) \in \mathbb{R}_{+}^{2}, \\
& 0 \leq a\left(y, y^{\prime}\right), b\left(y, y^{\prime}\right) \leq A(1+y)\left(1+y^{\prime}\right), \quad\left(y, y^{\prime}\right) \in \mathbb{R}_{+}^{2} .
\end{aligned}
$$

Note that (2.2) is physically natural for the coagulation rates and encompasses unbounded fragmentation rates. In fact, it can be slightly relaxed, see Section 6 . 
Under the sole bound (2.2) we do not know if the analysis presented herein is still valid. Furthermore it is an open problem to prove the existence of a solution to (1.1)-(1.2)-(1.3) under the two assumptions (2.1)-(2.2). One actually needs to make some additional structural assumptions on $a$ and $b[15,16,19]$, or to impose stronger growth conditions $[12,33]$. Here we relax the assumptions made in [33] and make the following growth assumption: for each $R \in \mathbb{R}_{+}$there holds

$$
\lim _{y^{\prime} \rightarrow+\infty} \sup _{y \in(0, R)} \frac{a\left(y, y^{\prime}\right)}{y^{\prime}}=\lim _{y^{\prime} \rightarrow+\infty} \sup _{y \in(0, R)} \frac{b\left(y, y^{\prime}\right)}{y^{\prime}}=0 .
$$

For example, (2.3) holds if the kinetic coefficients satisfy

$$
a\left(y, y^{\prime}\right), b\left(y, y^{\prime}\right) \leq A\left(1+y^{\alpha}\right)\left(1+\left(y^{\prime}\right)^{\alpha}\right)
$$

for some $A \in \mathbb{R}_{+}$and $\alpha \in[0,1)$.

We also assume

$$
f^{i n} \in L_{1}^{1}\left(\mathbb{R}_{+}\right):=L^{1}\left(\mathbb{R}_{+} ;(1+y) d y\right) \text { and is non-negative a.e. }
$$

We may now introduce the approximating DCF equations of the CCF equation. We fix $\varepsilon \in(0,1)$ and define the discrete kinetic coefficients $a_{i, j}^{\varepsilon}$ and $b_{i, j}^{\varepsilon}$ for $i, j \geq 1$ either by

$$
a_{i, j}^{\varepsilon}=\frac{1}{\varepsilon} \int_{\Lambda_{i}^{\varepsilon} \times \Lambda_{j}^{\varepsilon}} a\left(y, y^{\prime}\right) d y^{\prime} d y, \quad b_{i, j}^{\varepsilon}=\frac{1}{\varepsilon} \int_{\Lambda_{i}^{\varepsilon} \times \Lambda_{j}^{\varepsilon}} b\left(y, y^{\prime}\right) d y^{\prime} d y
$$

or by

$$
a_{i, j}^{\varepsilon}=\varepsilon a(\varepsilon i, \varepsilon j), \quad b_{i, j}^{\varepsilon}=\varepsilon b(\varepsilon i, \varepsilon j)
$$

if $a$ and $b$ are continuous functions. In both cases it readily follows from (2.1)-(2.2)(2.3) that the discrete kinetic coefficients satisfy the symmetry condition

$$
a_{i, j}^{\varepsilon}=a_{j, i}^{\varepsilon} \quad \text { and } \quad b_{i, j}^{\varepsilon}=b_{j, i}^{\varepsilon}, \quad i, j \in \mathbb{N} \backslash\{0\},
$$

and the growth conditions

$$
0 \leq a_{i j}^{\varepsilon}, b_{i j}^{\varepsilon} \leq A \varepsilon(1+\varepsilon i)(1+\varepsilon j), \quad i, j \geq 1,
$$

and

$$
\lim _{j \rightarrow+\infty} \frac{a_{i, j}^{\varepsilon}}{j}=\lim _{j \rightarrow+\infty} \frac{b_{i, j}^{\varepsilon}}{j}=0, \quad i \geq 1
$$

We also define the discrete initial data $c^{i n, \varepsilon}$ by

$$
c_{i}^{i n, \varepsilon}=\frac{1}{\varepsilon} \int_{\Lambda_{i}^{\varepsilon}} f^{i n}(y) d y, \quad i \geq 1
$$


Notice that, by (2.4), we have

$$
\varepsilon^{2} \sum_{i=1}^{\infty} i c_{i}^{i n, \varepsilon} \leq 2 \int_{0}^{\infty} f^{i n}(y) y d y
$$

and

$$
\varepsilon \sum_{i=1}^{\infty} c_{i}^{i n, \varepsilon} \leq \int_{0}^{\infty} f^{i n}(y) d y .
$$

We then consider a solution $c^{\varepsilon}=\left(c_{i}^{\varepsilon}\right)_{i \geq 1}$ to the DCF equation with kinetic coefficients $\left(a_{i, j}^{\varepsilon}\right)$ and $\left(b_{i, j}^{\varepsilon}\right)$ and initial datum $c_{i}^{i n, \varepsilon}$. As established in $[22,32]$ such a solution $c^{\varepsilon}$ exists and satisfies

$$
\sum_{i=1}^{\infty} i c_{i}^{\varepsilon}(t) \leq \sum_{i=1}^{\infty} i c_{i}^{i n, \varepsilon}, \quad t \geq 0
$$

We are now in a position to introduce the continuous formulation of the discrete quantities $c^{\varepsilon}, a_{i, j}^{\varepsilon}, b_{i, j}^{\varepsilon}$ and define the new functions $f_{\varepsilon}$ by (1.7) (with $c_{i}^{\varepsilon}$ instead of $c_{i}$ ) and $a_{\varepsilon}, b_{\varepsilon}$ by (1.8) (with $a_{i, j}^{\varepsilon}, b_{i, j}^{\varepsilon}$ instead of $a_{i, j}, b_{i, j}$ ). Notice that, with these notations, $a_{\varepsilon}$ and $b_{\varepsilon}$ satisfy (2.1) and the growth conditions (2.2) and (2.3) uniformly with respect to $\varepsilon \in(0,1)$. Namely, for any $R \in \mathbb{R}_{+}$, there exists a bounded function $\omega_{R}$ which decreases to zero as $y \rightarrow+\infty$ and such that

$$
\sup _{y^{\prime} \geq M} \sup _{y \in(0, R)} \frac{a^{\varepsilon}\left(y, y^{\prime}\right)}{y^{\prime}}+\sup _{y^{\prime} \geq M} \sup _{y \in(0, R)} \frac{b^{\varepsilon}\left(y, y^{\prime}\right)}{y^{\prime}} \leq \omega_{R}(M) \underset{M \rightarrow \infty}{\longrightarrow} 0
$$

and

$$
a_{\varepsilon} \rightarrow a, b_{\varepsilon} \rightarrow b \text { a.e. and weakly in } L_{\mathrm{loc}}^{\infty}\left(\mathbb{R}_{+}\right) .
$$

Notice also that $f_{\varepsilon}$ satisfies

$$
\int_{0}^{\infty} f_{\varepsilon}(t, y) d y=\varepsilon \sum_{i=1}^{\infty} c_{i}^{\varepsilon}(t) \text { and } \int_{0}^{\infty} f_{\varepsilon}(t, y) y d y=\varepsilon^{2} \sum_{i=1}^{\infty} i c_{i}^{\varepsilon}(t) .
$$

Before stating our result, let us make precise the notion of solution to (1.1) to be used in the sequel.

Definition 2.1 Assume that $a$ and $b$ satisfy (2.1)-(2.2). We say that $f=f(t, y)$ is a weak solution to the CCF equation (1.1)-(1.2)-(1.3) with the initial datum $f^{\text {in }}$ satisfying (2.4) if

$$
0 \leq f \in L^{\infty}\left(0, T ; L_{1}^{1}\left(\mathbb{R}_{+}\right)\right) \text {for each } T \in \mathbb{R}_{+}
$$

and (1.1) holds in $\mathcal{D}^{\prime}\left([0,+\infty) \times \mathbb{R}_{+}\right)$, that is,

$$
\int_{0}^{\infty} \int_{0}^{\infty} f \frac{\partial \psi}{\partial t} d y d t+\int_{0}^{\infty} f^{i n} \psi(0, .) d y=\int_{0}^{\infty} \int_{0}^{\infty} Q(f) \psi d y d t
$$

for any $\psi \in \mathcal{D}\left([0,+\infty) \times \mathbb{R}_{+}\right)$. 
Note that the boundedness assumptions (2.2) and (2.17) guarantee that the reaction terms $Q_{k}(f)$ belong to $L^{1}((0, T) \times(0, R))$ for any $k \in\{1, \ldots, 4\}, T \in \mathbb{R}_{+}$ and $R \in \mathbb{R}_{+}$. In particular the last term in (2.18) makes sense.

Another possible definition of solution is the following.

Definition 2.2 Assume that $a$ and $b$ satisfy (2.1)-(2.2). We say that $f=f(t, y)$ is a mild solution to the CCF equation (1.1)-(1.2)-(1.3) with the initial datum $f^{\text {in }}$ satisfying (2.4) if

$$
0 \leq f \in \mathcal{C}\left([0,+\infty) ; L^{1}\left(\mathbb{R}_{+}\right)\right) \cap L^{\infty}\left(0, T ; L_{1}^{1}\left(\mathbb{R}_{+}\right)\right) \text {for each } T \in \mathbb{R}_{+},
$$

with $f(0)=f^{\text {in }}$ and (1.1) holds in the mild sense : for $0 \leq t_{0}<t_{1}$ there holds

$$
f\left(t_{1}, .\right)-f\left(t_{0}, .\right)=\int_{t_{0}}^{t_{1}} Q(f(t, .)) d t \text { a.e. in } \mathbb{R}_{+} .
$$

Here again, (2.20) makes sense thanks to the bounds (2.2) and (2.19). At first glance it may seem that being a mild solution in the sense of Definition 2.2 is a stronger notion of solution than being a weak solution in the sense of Definition 2.1. In fact, we prove in the Appendix that these two notions are equivalent.

Our first result makes precise the connection between the DCF and $\mathrm{CCF}$ equations.

Theorem 2.3 Assume that $a$ and $b$ satisfy (2.1)-(2.2)-(2.3) and that $f^{\text {in }}$ satisfies (2.4). The family $\left(f_{\varepsilon}\right)$ of approximate solutions being defined above, there exists a weak solution $f$ to the CCF equation (1.1)-(1.2)-(1.3) with the initial datum fin such that, extracting a subsequence if necessary,

$$
f_{\varepsilon} \longrightarrow f \text { weakly in } L^{1}\left((0, T) \times \mathbb{R}_{+}\right) \text {for each } T \in \mathbb{R}_{+} \text {. }
$$

Obviously, if the weak solution to the CCF equation (1.1)-(1.2)-(1.3) is unique, it is the whole family $\left(f_{\varepsilon}\right)$ which converges. This is in particular the case when

$$
\begin{aligned}
& a\left(y, y^{\prime}\right) \leq K(1+y)^{1 / 2}\left(1+y^{\prime}\right)^{1 / 2} \\
& \int_{0}^{y}\left(1+y^{\prime}\right)^{1 / 2} b\left(y^{\prime}, y-y^{\prime}\right) d y^{\prime} \leq K(1+y)^{1 / 2}
\end{aligned}
$$

for $\left(y, y^{\prime}\right) \in \mathbb{R}_{+}^{2}$ and some constant $K>0$ [34]. Another uniqueness result may be found in $[12]$.

Remark 2.4 We have actually the stronger convergence

$$
f_{\varepsilon} \longrightarrow f \text { in } \mathcal{C}\left([0, T] ; w-L^{1}\left(\mathbb{R}_{+}\right)\right)
$$

for every $T \in \mathbb{R}_{+}$where $\mathcal{C}\left([0, T] ; w-L^{1}\left(\mathbb{R}_{+}\right)\right)$denotes the space of weakly continuous functions from $[0, T]$ in $L^{1}\left(\mathbb{R}_{+}\right)$. 
The conditions made on $a$ and $b$ may be relaxed in several directions and we refer to Section 6 for precise statements. A case of some particular interest is the case of sublinear coagulation coefficients, namely

$$
a\left(y, y^{\prime}\right) \leq A_{0}\left(1+y+y^{\prime}\right), \quad\left(y, y^{\prime}\right) \in \mathbb{R}_{+}^{2},
$$

for some $A_{0}>0$. In that case we have the following result.

Theorem 2.5 Assume that $a$ and $b$ satisfy (2.1), (2.2) and (2.22) and that $f^{\text {in }}$ satisfies (2.4). The kinetic coefficients $\left(a_{i, j}^{\varepsilon}\right)$ and $\left(b_{i, j}^{\varepsilon}\right)$ being still defined by (2.5) or (2.6), we denote by $c^{\varepsilon}$ a solution to the corresponding DCF equations (1.4)-(1.5) with initial datum $c^{i n, \varepsilon}=\left(c_{i}^{i n, \varepsilon}\right)$ given by (2.10) satisfying

$$
\sum_{i=1}^{\infty} i c_{i}^{\varepsilon}(t)=\sum_{i=1}^{\infty} i c_{i}^{i n, \varepsilon}, \quad t \in[0,+\infty)
$$

(the existence of such a solution follows from [4]). Putting

$$
f_{\varepsilon}(t, y)=\sum_{i=1}^{\infty} c_{i}^{\varepsilon}(t) \chi_{i}^{\varepsilon}(y), \quad(t, y) \in \mathbb{R}_{+}^{2},
$$

as before, there exists a weak solution $f$ to the CCF equation (1.1)-(1.2)-(1.3) with the initial datum fin such that, extracting a subsequence if necessary,

$$
f_{\varepsilon} \longrightarrow f \text { weakly in } L^{1}\left((0, T) \times \mathbb{R}_{+} ;(1+y) d t d y\right) \text { for each } T \in \mathbb{R}_{+}
$$

and

$$
\int_{0}^{\infty} f(t, y) y d y=\int_{0}^{\infty} f^{i n}(y) y d y \quad \text { for } t \geq 0
$$

Here again the convergence (2.24) may be improved to

$$
f_{\varepsilon} \longrightarrow f \text { in } \mathcal{C}\left([0, T] ; w-L^{1}\left(\mathbb{R}_{+} ;(1+y) d y\right)\right)
$$

for every $T \in \mathbb{R}_{+}$. A by-product of Theorem 2.5 is the existence of solutions to the CCF equation satisfying (2.25) under the only assumption (2.4) on the initial data $f^{i n}$. Stronger assumptions on $f^{\text {in }}$ are required in the analysis of [12] and Theorem 2.5 thus extends the results of [12] for fragmentation coefficients satisfying simultaneously (2.2) and the growth conditions of [12].

\section{3 a priori estimates}

In this section we consider the family of discrete kinetic coefficients $\left(a_{i, j}^{\varepsilon}\right)$ and $\left(b_{i, j}^{\varepsilon}\right)$ defined by (2.5) or (2.6). We are going to establish several estimates on $f_{\varepsilon}$ defined by (1.7) which are uniform with respect to $\varepsilon>0$ and ultimately imply that $\left(f_{\varepsilon}\right)$ lies 
in a weakly compact set of $L^{1}$. Let us emphasize here that the estimates derived in this section are valid under the sole assumptions (2.1) and (2.2) and in particular do not rely on (2.3). We put

$$
M=\int_{0}^{\infty} f^{i n}(y)(1+y) d y .
$$

In the following we denote by $C$ any positive constant depending only on $A$ and $M$. The dependence of $C$ upon additional parameters will be indicated explicitly.

Lemma 3.1 For any $t \geq 0$ there holds

$$
\int_{0}^{\infty} f_{\varepsilon}(t, y) y d y \leq 2 M
$$

Proof. By (2.11), (2.13) and (2.16) we have

$$
\int_{0}^{\infty} f_{\varepsilon}(t, y) y d y=\varepsilon^{2} \sum_{i=1}^{\infty} i c_{i}^{\varepsilon}(t) \leq \varepsilon^{2} \sum_{i=1}^{\infty} i c_{i}^{i n, \varepsilon} \leq 2 \int_{0}^{\infty} f^{i n}(y) y d y
$$

whence (3.1).

Lemma 3.2 For $T \in \mathbb{R}_{+}, R \in \mathbb{R}_{+}$and $t \in[0, T]$ we have

$$
\int_{0}^{R} f_{\varepsilon}(t, y) d y \leq C(T, R)
$$

Proof. Let $m$ be the integer such that $m \varepsilon<R \leq(m+1) \varepsilon$. We infer from (1.6) and the non-negativity of $b_{i, j}^{\varepsilon}$ and $c^{\varepsilon}$ that

$$
\begin{aligned}
\frac{d}{d t} \sum_{i=1}^{m+1} \varepsilon c_{i}^{\varepsilon} & \leq \frac{\varepsilon}{2} \sum_{i, j=1}^{\infty} a_{i, j}^{\varepsilon} c_{i}^{\varepsilon} c_{j}^{\varepsilon}\left(\mathbf{1}_{i+j \leq m+1}-\mathbf{1}_{i \leq m+1}-\mathbf{1}_{j \leq m+1}\right) \\
& +\varepsilon \sum_{i=1}^{m+1} \sum_{j=1}^{\infty} b_{i, j}^{\varepsilon} c_{i+j}^{\varepsilon} \\
& \leq \sum_{k=1}^{\infty} \varepsilon c_{k}^{\varepsilon} \sum_{i=1}^{m+1} b_{i, k-i}^{\varepsilon} .
\end{aligned}
$$

It follows from (2.8) that, for any $k \geq 2$,

$$
\sum_{i=1}^{m+1} b_{i, k-i}^{\varepsilon} \leq A \sum_{i=1}^{m+1} \varepsilon(1+\varepsilon i)(1+\varepsilon k) \leq C_{R}(1+\varepsilon k) .
$$

Therefore we have

$$
\frac{d}{d t} \sum_{i=1}^{m+1} \varepsilon c_{i}^{\varepsilon} \leq C_{R} \sum_{k=1}^{\infty} \varepsilon c_{k}^{\varepsilon}(1+\varepsilon k) \leq C_{R}\left(\sum_{i=1}^{m+1} \varepsilon c_{i}^{\varepsilon}+2 M\right) .
$$


Using the Gronwall Lemma yields that

$$
\sum_{i=1}^{m+1} \varepsilon c_{i}^{\varepsilon}(t) \leq C(R, T) \sum_{i=1}^{m+1} \varepsilon c_{i}^{i n, \varepsilon}
$$

for any $t \in[0, T]$ and we conclude thanks to (2.12) and (2.16).

Lemma 3.3 Let $\Phi \in \mathcal{C}^{1}([0,+\infty))$ be a non-negative convex function such that $\Phi(0)=0, \Phi^{\prime}(0)=1$ and $\Phi^{\prime}$ is concave. If

$$
L_{\Phi}:=\int_{0}^{\infty} \Phi\left(f^{i n}\right)(y) d y<\infty
$$

there holds

$$
\int_{0}^{R} \Phi\left(f_{\varepsilon}(t, y)\right) d y \leq C(T, R)\left(L_{\Phi}+\Phi(1)\right)
$$

for each $t \in[0, T], T \in \mathbb{R}_{+}$and $R \in \mathbb{R}_{+}$.

Proof. We first recall that the properties of $\Phi$ imply that

$$
u \Phi^{\prime}(v) \leq \Phi(u)+\Phi(v), \quad u, v \geq 0
$$

Indeed, owing to the convexity of $\Phi$ and the concavity of $\Phi^{\prime}$, we have $v \Phi^{\prime}(v) \leq 2 \Phi(v)$ by [20, Lemma A.1] and the convexity of $\Phi$ further entails that

$$
u \Phi^{\prime}(v) \leq \Phi(u)-\Phi(v)+v \Phi^{\prime}(v) \leq \Phi(u)+\Phi(v) .
$$

We denote again by $m$ the integer such that $m \varepsilon<R \leq(m+1) \varepsilon$. We infer from (1.4)-(1.5), (3.6) and the non-negativity of $a_{i, j}^{\varepsilon}, b_{i, j}^{\varepsilon}$ and $c^{\varepsilon}$ that

$$
\begin{aligned}
\frac{d}{d t} \sum_{i=1}^{m+1} \varepsilon \Phi\left(c_{i}^{\varepsilon}\right) \leq & \varepsilon \sum_{i=1}^{m+1}\left(Q_{1, i}^{\varepsilon}\left(c^{\varepsilon}\right)+Q_{4, i}^{\varepsilon}\left(c^{\varepsilon}\right)\right) \Phi^{\prime}\left(c_{i}^{\varepsilon}\right) \\
\leq & \frac{\varepsilon}{2} \sum_{i=1}^{m+1} \sum_{j=1}^{i-1} a_{i-j, j}^{\varepsilon} c_{i-j}^{\varepsilon} c_{j}^{\varepsilon} \Phi^{\prime}\left(c_{i}^{\varepsilon}\right)+\varepsilon \sum_{i=1}^{m+1} \sum_{j=1}^{\infty} b_{i, j}^{\varepsilon} c_{i+j}^{\varepsilon} \Phi^{\prime}\left(c_{i}^{\varepsilon}\right) \\
\leq & \frac{\varepsilon}{2} \sum_{i=1}^{m+1} \sum_{j=1}^{i-1} a_{i-j, j}^{\varepsilon}\left(\Phi\left(c_{i-j}^{\varepsilon}\right)+\Phi\left(c_{i}^{\varepsilon}\right)\right) c_{j}^{\varepsilon} \\
& +\varepsilon \sum_{k=2}^{\infty} c_{k}^{\varepsilon} \sum_{i=1}^{m+1} b_{i, k-i}^{\varepsilon}\left(\Phi(1)+\Phi\left(c_{i}^{\varepsilon}\right)\right) .
\end{aligned}
$$

Since we have

$$
\sup _{i, j \leq m+1} a_{i, j}^{\varepsilon} \leq C(R) \varepsilon \text { and } \sup _{i \leq m+1} b_{i, k-i}^{\varepsilon} \leq C(R) \varepsilon(1+\varepsilon k)
$$


by (2.8) for any $k \geq 2$, we get

$$
\begin{aligned}
\frac{d}{d t} \sum_{i=1}^{m+1} \varepsilon \Phi\left(c_{i}^{\varepsilon}\right) \leq & C(R)\left(\sum_{i=1}^{m+1} \varepsilon \Phi\left(c_{i}^{\varepsilon}\right)\right)\left(\sum_{j=1}^{m+1} \varepsilon c_{j}^{\varepsilon}\right) \\
& +C(R)\left(\sum_{k=1}^{\infty} \varepsilon(1+\varepsilon k) c_{k}^{\varepsilon}\right)\left(\sum_{i=1}^{m+1} \varepsilon\left(\Phi(1)+\Phi\left(c_{i}^{\varepsilon}\right)\right)\right) .
\end{aligned}
$$

Therefore, using Lemma 3.2 (and more precisely (3.3)), (2.16) and (3.2) we obtain

$$
\frac{d}{d t} \sum_{i=1}^{m+1} \varepsilon \Phi\left(c_{i}^{\varepsilon}\right) \leq C(T, R)\left(\sum_{i=1}^{m+1} \varepsilon \Phi\left(c_{i}^{\varepsilon}\right)+(R+1) \Phi(1)\right)
$$

for any $t \in[0, T]$, from which we deduce

$$
\sum_{i=1}^{m+1} \varepsilon \Phi\left(c_{i}^{\varepsilon}(t)\right) \leq C(T, R)\left(\Phi(1)+\sum_{i=1}^{m+1} \varepsilon \Phi\left(c_{i}^{i n, \varepsilon}\right)\right)
$$

by the Gronwall Lemma. Finally the Jensen inequality ensures that

$$
\sum_{i=1}^{m+1} \varepsilon \Phi\left(c_{i}^{i n, \varepsilon}\right) \leq \sum_{i=1}^{m+1} \int_{\Lambda_{i}^{\varepsilon}} \Phi\left(f^{i n}(y)\right) d y \leq L_{\Phi}
$$

and thus,

$$
\int_{0}^{R} \Phi\left(f_{\varepsilon}(t, y)\right) d y \leq \varepsilon \sum_{i=1}^{m+1} \Phi\left(c_{i}^{\varepsilon}(t)\right) \leq C(T, R)\left(\Phi(1)+L_{\Phi}\right),
$$

which completes the proof of (3.5).

Lemma 3.4 There exists $f \in L_{\text {loc }}^{\infty}\left([0,+\infty) ; L^{1}\left(\mathbb{R}_{+}\right)\right.$) and a subsequence of $f_{\varepsilon}$ (not relabeled) such that, for any $T \in \mathbb{R}_{+}$,

$$
f_{\varepsilon} \rightarrow f \quad \text { weakly in } L^{1}\left((0, T) \times \mathbb{R}_{+}\right) .
$$

Proof. Since $f^{i n} \in L^{1}\left(\mathbb{R}_{+}\right)$it follows from a refined version of the de la ValléePoussin theorem $[9,23]$ that there exists a function $\Phi$ fulfilling the assumptions of Lemma 3.3 and such that $\Phi(r) / r \rightarrow+\infty$ as $r \rightarrow+\infty$ and

$$
\int_{\mathbb{R}_{+}} \Phi\left(f^{i n}\right) d y<\infty
$$

Gathering Lemma 3.1, Lemma 3.2 and Lemma 3.3 we conclude that

$$
\sup _{\varepsilon>0} \sup _{[0, T]}\left\{\int_{0}^{\infty}(1+y) f_{\varepsilon} d y+\int_{0}^{R} \Phi\left(f_{\varepsilon}\right) d y\right\}<\infty
$$


for any $R \in \mathbb{R}_{+}$and $T \in \mathbb{R}_{+}$, which implies that $\left(f_{\varepsilon}\right)$ lies in a weakly compact subset of $L^{1}((0, T) \times(0, R))$ by the Dunford-Pettis theorem. We deduce that there exists $f \geq 0$ such that (3.7) holds true. Moreover, thanks to $(3.8), f \in L^{\infty}\left(0, T ; L_{1}^{1}\left(\mathbb{R}_{+}\right)\right)$.

We now state a fourth a priori bound which will be useful to pass to the limit in the (quadratic) coagulation terms.

Lemma 3.5 For any $T \in \mathbb{R}_{+}, R \in \mathbb{R}_{+}$and $\psi \in L^{\infty}(0, R)$

$$
\int_{0}^{R} f_{\varepsilon}(., y) \psi(y) d y \text { is bounded in } W^{1, \infty}(0, T) .
$$

Proof. We define the sequence $\psi_{i}^{\varepsilon}$ from $\psi$ by (1.10) and denote by $m$ the integer such that $m \varepsilon \leq R<(m+1) \varepsilon$. We infer from (2.8) that

$$
\begin{aligned}
& \left|\frac{d}{d t} \int_{0}^{R} f_{\varepsilon}(t, y) \psi(y) d y\right|=\varepsilon\left|\frac{d}{d t} \sum_{i=1}^{m+1} c_{i}^{\varepsilon} \psi_{i}^{\varepsilon}\right| \leq \varepsilon \sum_{i=1}^{m+1}\left|Q_{i}\left(c^{\varepsilon}\right) \psi_{i}^{\varepsilon}\right| \\
& \quad \leq \frac{3 \varepsilon}{2}\|\psi\|_{L^{\infty}}\left(\sum_{i=1}^{m+1} \sum_{j=1}^{\infty} a_{i, j}^{\varepsilon} c_{i}^{\varepsilon} c_{j}^{\varepsilon}+\sum_{k=1}^{\infty} c_{k}^{\varepsilon} \sum_{\ell=1}^{m+1} b_{k-\ell, \ell}^{\varepsilon}\right) \\
& \quad \leq \frac{3 A}{2}\|\psi\|_{L^{\infty}}\left\{\left(\sum_{i=1}^{\infty} \varepsilon(1+\varepsilon i) c_{i}^{\varepsilon}\right)^{2}+\sum_{k=1}^{\infty} \varepsilon(1+\varepsilon k) c_{k}^{\varepsilon}\left(\sum_{\ell=1}^{m+1}(1+\varepsilon \ell) \varepsilon\right)\right\}
\end{aligned}
$$

and the right-hand side of the above inequality is bounded in $L^{\infty}(0, T)$ by Lemma 3.1, Lemma 3.2 and (2.16).

Combining Lemma 3.5 and (3.8) allows us to obtain the convergence of $\left(f^{\varepsilon}\right)$ towards $f$ in a stronger topology. Notice however that this stronger convergence will not be used in the proof of Theorem 2.3.

Corollary 3.6 The convergence (3.7) may be improved to

$$
f_{\varepsilon} \rightarrow f \quad \text { in } \quad \mathcal{C}\left([0, T] ; w-L^{1}\left(\mathbb{R}_{+}\right)\right) .
$$

Proof. Fix $R \in \mathbb{R}_{+}$. On the one hand, $\left(f_{\varepsilon}(t)\right)$ lies in a weakly compact set of $L^{1}(0, R)$ by (3.8). On the other hand, Lemma 3.5 ensures that $\left(f_{\varepsilon}\right)$ is weakly equicontinuous in $L^{1}(0, R)$ at every $t \in[0, T]$ (in the sense of [35, Definition 1.3.1]). Consequently, according to a variant of the Arzelà-Ascoli theorem (see, e.g., [35, Theorem 1.3.2]), the sequence $\left(f_{\varepsilon}\right)$ is relatively compact in $\mathcal{C}\left([0, T] ; w-L^{1}(0, R)\right)$. This last fact and (3.8) then entail (3.10). 


\section{Passing to the limit}

The proof of Theorem 2.3 is splitted in two steps. We consider a function $\varphi \in$ $\mathcal{D}\left([0,+\infty) \times \mathbb{R}_{+}\right)$and define the $\varepsilon$-step function $\varphi_{\varepsilon}(t)$ by (1.10) for each $t \geq 0$. We first infer from (1.4) and (1.5) that $f_{\varepsilon}$ satisfies the following equation

$$
\begin{aligned}
& \int_{0}^{\infty} \int_{0}^{\infty} f_{\varepsilon} \frac{\partial \varphi_{\varepsilon}}{\partial t} d y d t+\int_{0}^{\infty} f_{\varepsilon}(0) \varphi_{\varepsilon}(0) d y \\
= & -\frac{1}{2} \int_{0}^{\infty} \int_{0}^{\infty} \int_{0}^{\infty}\left(a_{\varepsilon} f_{\varepsilon} f_{\varepsilon}^{\prime}-b_{\varepsilon} T_{\varepsilon}\left(f_{\varepsilon}\right)\right)\left(T_{\varepsilon}\left(\varphi_{\varepsilon}\right)-\varphi_{\varepsilon}-\varphi_{\varepsilon}^{\prime}\right) d y d y^{\prime} d t .
\end{aligned}
$$

Step 1: Convergence results. We prove several convergence results which will be needed to pass to the limit in (4.1). We begin with two elementary convergence results for test functions.

Lemma 4.1 The sequence $\left(\varphi_{\varepsilon}\right)$ satisfies

$$
\begin{aligned}
& \varphi_{\varepsilon} \rightarrow \varphi \text { strongly in } L^{\infty}\left(\mathbb{R}_{+}^{2}\right), \\
& T_{\varepsilon}\left(\varphi_{\varepsilon}\right) \rightarrow\left\{\left(t, y, y^{\prime}\right) \mapsto \varphi\left(t, y+y^{\prime}\right)\right\} \text { strongly in } L^{\infty}\left([0,+\infty) \times \mathbb{R}_{+}^{2}\right) .
\end{aligned}
$$

Proof. For any fixed $t, y, y^{\prime} \in \mathbb{R}_{+}$we have

$$
\begin{aligned}
& \left|T_{\varepsilon}\left(\varphi_{\varepsilon}\right)\left(t, y, y^{\prime}\right)-\varphi\left(t, y+y^{\prime}\right)\right| \\
= & \left|\sum_{i, j=1}^{\infty} \chi_{i}^{\varepsilon}(y) \chi_{j}^{\varepsilon}\left(y^{\prime}\right) \frac{1}{\varepsilon} \int_{\Lambda_{i+j}^{\varepsilon}}\left(\varphi(t, z)-\varphi\left(t, y+y^{\prime}\right)\right) d z\right| \\
\leq & \sup _{\left|y+y^{\prime}-z\right| \leq 2 \varepsilon}\left|\varphi(t, z)-\varphi\left(t, y+y^{\prime}\right)\right| \\
\leq & 2 \varepsilon \sup _{\mathbb{R}_{+}^{2}}\left|\frac{\partial \varphi}{\partial z}(t, z)\right|
\end{aligned}
$$

and (4.3) follows. Assertion (4.2) may be proved in the same way.

Lemma 4.2 For any $\psi \in C_{c}^{1}\left(\mathbb{R}_{+}^{3}\right)$ we define two functions $S_{\varepsilon}(\psi)$ and $S(\psi)$ in $L^{\infty}\left(\mathbb{R}_{+}^{2}\right)$ by

$$
\begin{aligned}
S_{\varepsilon}(\psi)(t, z) & :=\sum_{k=2}^{\infty} \chi_{k}^{\varepsilon}(z) \sum_{j=1}^{k-1} \frac{1}{\varepsilon} \iint_{\Lambda_{k-j}^{\varepsilon} \times \Lambda_{j}^{\varepsilon}} \psi\left(t, y, y^{\prime}\right) d y d y^{\prime} \\
S(\psi)(t, z) & :=\int_{0}^{z} \psi\left(t, z-y^{\prime}, y^{\prime}\right) d y^{\prime} .
\end{aligned}
$$

There holds

$$
S_{\varepsilon}(\psi) \underset{\varepsilon \rightarrow 0}{\longrightarrow} S(\psi) \text { strongly in } L^{\infty}\left(\mathbb{R}_{+}^{2}\right)
$$


Proof. Fix $(t, z) \in \mathbb{R}_{+}^{2}, \varepsilon>0$ and let $k$ be the integer such that $(k-1 / 2) \varepsilon \leq z \leq$ $(k+1 / 2) \varepsilon$. Either $k=0$ or $k=1$ and

$$
\left|\left(S_{\varepsilon}(\psi)-S(\psi)\right)(t, z)\right|=|S(\psi)(t, z)| \leq 2 \varepsilon\|\psi\|_{L^{\infty}} .
$$

Or $k \geq 2$ and we easily compute

$$
\begin{aligned}
\left(S(\psi)-S_{\varepsilon}(\psi)\right)(t, z) & =\int_{0}^{z} \psi(t, z-y, y) d y-\sum_{j=1}^{k-1} \frac{1}{\varepsilon} \iint_{\Lambda_{k-j}^{\varepsilon} \times \Lambda_{j}^{\varepsilon}} \psi\left(t, y, y^{\prime}\right) d y d y^{\prime} \\
& =\int_{0}^{\varepsilon / 2} \psi(t, z-y, y) d y+\int_{(k-1 / 2) \varepsilon}^{z} \psi(t, z-y, y) d y \\
& +\sum_{j=1}^{k-1} \int_{\Lambda_{j}^{\varepsilon}}(\psi(t, z-y, y)-\psi(t,(k-j) \varepsilon, \varepsilon j)) d y \\
& +\sum_{j=1}^{k-1} \frac{1}{\varepsilon} \iint_{\Lambda_{k-j}^{\varepsilon} \times \Lambda_{j}^{\varepsilon}}\left(\psi(t,(k-j) \varepsilon, \varepsilon j)-\psi\left(t, y, y^{\prime}\right)\right) d y d y^{\prime}
\end{aligned}
$$

This implies that

$$
\sup _{(t, z) \in \mathbb{R}_{+}^{2} z}\left|\left(S(\psi)-S_{\varepsilon}(\psi)\right)(t, z)\right| \leq\left(2\|\psi\|_{L^{\infty}}+2 L\|\nabla \psi\|_{L^{\infty}}\right) \varepsilon
$$

where $L \geq 1$ is such that $\operatorname{supp} \psi \subset[0, L-1]^{3}$. Then (4.6) readily follows.

Let us recall the following lemma which is a classical consequence of the Egorov and Dunford-Pettis theorems (see, e.g., [22, Lemma A.2] for a proof).

Lemma 4.3 Let $U$ be an open subset of $\mathbb{R}^{m}, m \geq 1$, and consider two sequences $\left(v_{n}\right)$ in $L^{1}(U)$ and $\left(w_{n}\right)$ in $L^{\infty}(U)$ and two functions $v \in L^{1}(U)$ and $w \in L^{\infty}(U)$ such that

$$
\begin{aligned}
& v_{n} \rightarrow v \quad \text { weakly in } L^{1}(U), \\
& \left|w_{n}\right| \leq C \text { and } w_{n} \rightarrow w \quad \text { a.e. }
\end{aligned}
$$

for some $C \geq 0$. Then

$$
\lim _{n \rightarrow \infty}\left\|v_{n}\left(w_{n}-w\right)\right\|_{L^{1}}=0 \quad \text { and } \quad v_{n} w_{n} \rightarrow v w \quad \text { weakly in } L^{1}(U) .
$$

We are now in a position to prove convergence results for the sequence $\left(f_{\varepsilon}\right)$.

Lemma 4.4 The sequence $\left(f_{\varepsilon}\right)$ satisfies: for any $T \in \mathbb{R}_{+}$and $R \geq 1$ we have

$$
\begin{aligned}
& f_{\varepsilon}(t, y) f_{\varepsilon}\left(t, y^{\prime}\right) \rightarrow f(t, y) f\left(t, y^{\prime}\right) \text { weakly in } L^{1}\left((0, T) \times(0, R)^{2}\right), \\
& T_{\varepsilon}\left(f_{\varepsilon}\right)\left(t, y, y^{\prime}\right) \rightarrow f\left(t, y+y^{\prime}\right) \text { weakly in } L^{1}\left((0, T) \times(0, R)^{2}\right), \\
& \sup _{[0, T]} \int_{0}^{R}\left(\int_{0}^{\infty} y^{\prime} T_{\varepsilon}\left(f_{\varepsilon}\right) d y^{\prime}\right) d y \leq C(T, R) .
\end{aligned}
$$


Proof. Step 1. We prove (4.8). On the one hand, let $\Phi$ be the function introduced in the proof of Lemma 3.4. Denoting by $m$ the integer such that $R \in \Lambda_{m}^{\varepsilon}$ we have for any $t \in[0, T]$

$$
\begin{aligned}
\int_{0}^{R} \int_{0}^{R} \Phi\left(T_{\varepsilon}\left(f_{\varepsilon}\right)\right) d y^{\prime} d y & =\sum_{1 \leq i, j \leq m} \Phi\left(c_{i+j}^{\varepsilon}\right) \varepsilon^{2} \leq \sum_{k=2}^{2 m} \sum_{j=1}^{m} \Phi\left(c_{k}^{\varepsilon}\right) \varepsilon^{2} \\
& \leq(R+1) \sum_{k=2}^{2 m} \Phi\left(c_{k}^{\varepsilon}\right) \varepsilon \leq C(R) \int_{0}^{2(R+1)} \Phi\left(f_{\varepsilon}\right) d y \\
& \leq C(R, T) \int_{0}^{2(R+1)} \Phi\left(f^{i n}\right) d y .
\end{aligned}
$$

Since $\Phi(r) / r \rightarrow+\infty$ as $r \rightarrow+\infty$ the above estimate implies that $\left(T_{\varepsilon}\left(f_{\varepsilon}\right)\right)$ lies in a weakly compact subset of $L^{1}\left((0, T) \times(0, R)^{2}\right)$.

On the other hand, fix $\psi \in \mathcal{D}\left(\mathbb{R}_{+}^{3}\right)$ and compute

$$
\begin{aligned}
\int_{0}^{\infty} \int_{0}^{\infty} \int_{0}^{\infty} T_{\varepsilon}\left(f_{\varepsilon}\right) \psi d y^{\prime} d y d t & =\int_{0}^{\infty} \sum_{i, j=1}^{\infty} c_{i+j}^{\varepsilon} \iint_{\Lambda_{i}^{\varepsilon} \times \Lambda_{j}^{\varepsilon}} \psi\left(t, y, y^{\prime}\right) d y^{\prime} d y d t \\
& =\int_{0}^{\infty} \sum_{k=2}^{\infty} \varepsilon c_{k}^{\varepsilon} \sum_{j=1}^{k-1} \frac{1}{\varepsilon} \iint_{\Lambda_{k-j}^{\varepsilon} \times \Lambda_{j}^{\varepsilon}} \psi\left(t, y, y^{\prime}\right) d y^{\prime} d y d t \\
& =\int_{0}^{\infty} \int_{0}^{\infty} f_{\varepsilon} S_{\varepsilon}(\psi) d z d t,
\end{aligned}
$$

where $S_{\varepsilon}(\psi)$ is defined by (4.4). ¿From Lemma 3.4, Lemma 4.2 and Lemma 4.3 we deduce that

$$
\int_{0}^{\infty} \int_{0}^{\infty} f_{\varepsilon} S_{\varepsilon}(\psi) d z d t \underset{\varepsilon \rightarrow 0}{\longrightarrow} \int_{0}^{\infty} \int_{0}^{\infty} f S(\psi) d z d t
$$

where $S(\psi)$ is defined by (4.5). Finally, combining (4.10), (4.11) and the change of variables $\left(y, y^{\prime}\right) \rightarrow\left(y, z=y+y^{\prime}\right)$, we get

$$
\int_{0}^{\infty} \int_{0}^{\infty} \int_{0}^{\infty} T_{\varepsilon}\left(f_{\varepsilon}\right) \psi d y^{\prime} d y d t \underset{\varepsilon \rightarrow 0}{\longrightarrow} \int_{0}^{\infty} \int_{0}^{\infty} \int_{0}^{\infty} f\left(t, y+y^{\prime}\right) \psi\left(t, y, y^{\prime}\right) d y^{\prime} d y d t .
$$

This exactly means that $T_{\varepsilon}\left(f_{\varepsilon}\right) \rightarrow f\left(y+y^{\prime}\right)$ in the sense of distributions and therefore (4.8) follows from the weak $L^{1}$-compactness previously established.

Step 2. We prove (4.9). Let $m$ be the integer such that $R \in \Lambda_{m+1}^{\varepsilon}$. We just have to compute

$$
\begin{gathered}
\int_{0}^{R}\left(\int_{0}^{\infty} y^{\prime} T_{\varepsilon}\left(f_{\varepsilon}\right) d y^{\prime}\right) d y \leq \sum_{i=1}^{m+1} \sum_{j=1}^{\infty} \iint_{\Lambda_{i}^{\varepsilon} \times \Lambda_{j}^{\varepsilon}} y^{\prime} T_{\varepsilon}\left(f_{\varepsilon}\right)\left(y, y^{\prime}\right) d y d y^{\prime} \\
\leq \sum_{i=1}^{m+1} \sum_{j=1}^{\infty} \varepsilon(j+i) c_{i+j}^{\varepsilon} \varepsilon^{2} \leq \sum_{k=1}^{\infty} \varepsilon^{2} k c_{k}^{\varepsilon} \sum_{i=1}^{m+1} \varepsilon \\
\leq(R+1) \int_{0}^{\infty} y f_{\varepsilon} d y \leq C(T, R)
\end{gathered}
$$


for any $t \in[0, T]$ by Lemma 3.1.

Step 3. We prove (4.7). We first claim that, for any $\psi \in L^{\infty}\left((0, T) \times(0, R)^{2}\right)$, there holds

$$
\int_{0}^{R} f_{\varepsilon}^{\prime} \psi^{\prime} d y^{\prime} \underset{\varepsilon \rightarrow 0}{\longrightarrow} \int_{0}^{R} f^{\prime} \psi^{\prime} d y^{\prime} \text { strongly in } L^{1}((0, T) \times(0, R)) .
$$

Indeed, from Lemma 3.5, we know that (4.12) holds true for any function $\psi$ of the form

$$
\psi\left(t, y, y^{\prime}\right)=\sum_{n=1}^{N} u_{n}\left(y^{\prime}\right) v_{n}(t, y)
$$

Now, if $\psi$ is an arbitrary function in $L^{\infty}\left((0, T) \times(0, R)^{2}\right)$ there exists a sequence $\left(\psi_{\alpha}\right)$ of functions of the form (4.13) such that $\psi_{\alpha} \rightarrow \psi$ a.e. and weakly in $L^{\infty}((0, T) \times$ $\left.(0, R)^{2}\right)$. Therefore,

$$
\begin{aligned}
\left\|\int_{0}^{R}\left(f_{\varepsilon}^{\prime}-f^{\prime}\right) \psi^{\prime} d y^{\prime}\right\|_{L^{1}} \leq & \left\|\int_{0}^{R}\left(f_{\varepsilon}^{\prime}-f^{\prime}\right) \psi_{\alpha}^{\prime} d y^{\prime}\right\|_{L^{1}} \\
& +\sup _{\varepsilon} \int_{0}^{T} \int_{0}^{R} \int_{0}^{R}\left|f_{\varepsilon}^{\prime}-f^{\prime}\right|\left|\psi^{\prime}-\psi_{\alpha}^{\prime}\right| d y^{\prime} d y d t,
\end{aligned}
$$

whence

$$
\limsup _{\varepsilon \rightarrow 0}\left\|\int_{0}^{R}\left(f_{\varepsilon}^{\prime}-f^{\prime}\right) \psi^{\prime} d y^{\prime}\right\|_{L^{1}} \leq \sup _{\varepsilon} \int_{0}^{T} \int_{0}^{R} \int_{0}^{R}\left|f_{\varepsilon}^{\prime}-f^{\prime}\right|\left|\psi^{\prime}-\psi_{\alpha}^{\prime}\right| d y^{\prime} d y d t
$$

for every $\alpha>0$. A variant of Lemma 4.3 (see, e.g., [22, Lemma A.1]) then ensures that the right-hand side of the above equation converges to zero as $\alpha \rightarrow 0$ and thus (4.12) holds true for any $\psi \in L^{\infty}\left((0, T) \times(0, R)^{2}\right)$. Now, for a given $\psi \in$ $L^{\infty}\left((0, T) \times(0, R)^{2}\right)$, it follows from $(3.2),(4.12)$ and Lemma 4.3 that

$$
\int_{0}^{T} \int_{0}^{R} f_{\varepsilon}\left(\int_{0}^{R} f_{\varepsilon}^{\prime} \psi d y^{\prime}\right) d y d t \underset{\varepsilon \rightarrow 0}{\longrightarrow} \int_{0}^{T} \int_{0}^{R} f\left(\int_{0}^{R} f^{\prime} \psi d y^{\prime}\right) d y d t
$$

which exactly means that (4.7) holds.

Step 2: Passing to the limit in (4.1). Thanks to the previous analysis we now have all the necessary tools to pass to the limit in (4.1). We consider $\varphi \in \mathcal{D}\left([0,+\infty) \times \mathbb{R}_{+}\right)$ with $\operatorname{supp} \varphi \subset[0, L-1]^{2}$ for some $L \geq 1$ and recall that $\varphi_{\varepsilon}(t)$ is the $\varepsilon$-step function defined by (1.10) for $t \geq 0$. Let $T \in \mathbb{R}_{+}$and $R \in \mathbb{R}_{+}$. On the one hand we have

$$
\begin{gathered}
\int_{0}^{T} \int_{0}^{R} \int_{0}^{R}\left[a_{\varepsilon} f_{\varepsilon} f_{\varepsilon}^{\prime}-b_{\varepsilon} T_{\varepsilon}\left(f_{\varepsilon}\right)\right]\left[T_{\varepsilon}\left(\varphi_{\varepsilon}\right)-\varphi_{\varepsilon}-\varphi_{\varepsilon}^{\prime}\right] d y d y^{\prime} d t \\
\underset{\varepsilon \rightarrow 0}{\longrightarrow} \int_{0}^{T} \int_{0}^{R} \int_{0}^{R}\left[a f f^{\prime}-b f^{\prime \prime}\right]\left[\varphi^{\prime \prime}-\varphi-\varphi^{\prime}\right] d y d y^{\prime} d t
\end{gathered}
$$

where we have used (2.15), Lemma 4.1, Lemma 4.4 and Lemma 4.3 with first $v_{\varepsilon}=$ $f_{\varepsilon} f_{\varepsilon}^{\prime}, w_{\varepsilon}=a_{\varepsilon}\left[T_{\varepsilon}\left(\varphi_{\varepsilon}\right)-\varphi_{\varepsilon}-\varphi_{\varepsilon}^{\prime}\right]$ and next $v_{\varepsilon}=T_{\varepsilon}\left(f_{\varepsilon}\right), w_{\varepsilon}=b_{\varepsilon}\left[T_{\varepsilon}\left(\varphi_{\varepsilon}\right)-\varphi_{\varepsilon}-\varphi_{\varepsilon}^{\prime}\right]$. 
On the other hand, for $R \geq L$,

$$
\begin{aligned}
& \left|\iint_{\mathbb{R}_{+}^{2} \backslash[0, R]^{2}}\left(b_{\varepsilon} T_{\varepsilon}\left(f_{\varepsilon}\right)-a_{\varepsilon} f_{\varepsilon} f_{\varepsilon}^{\prime}\right)\left(\varphi_{\varepsilon}+\varphi_{\varepsilon}^{\prime}\right) d y d y^{\prime}\right| \\
& \leq 2 \int_{0}^{L}\left(\int_{R}^{\infty} a_{\varepsilon} f_{\varepsilon} f_{\varepsilon}^{\prime} \varphi_{\varepsilon} d y^{\prime}\right) d y+2 \int_{0}^{L}\left(\int_{R}^{\infty} b_{\varepsilon} T_{\varepsilon}\left(f_{\varepsilon}\right) \varphi_{\varepsilon} d y^{\prime}\right) d y \\
& \leq 2 \sup _{y \leq L, y^{\prime} \geq R}\left|\frac{a_{\varepsilon}\left(y, y^{\prime}\right)}{y^{\prime}}\right|\|\varphi\|_{L^{\infty}} \int_{0}^{\infty} f_{\varepsilon} d y \int_{0}^{\infty} y^{\prime} f_{\varepsilon}^{\prime} d y^{\prime} \\
& \quad+2 \sup _{y \leq L, y^{\prime} \geq R}\left|\frac{b_{\varepsilon}\left(y, y^{\prime}\right)}{y^{\prime}}\right|\|\varphi\|_{L^{\infty}} \int_{0}^{L} \int_{0}^{\infty} y^{\prime} T_{\varepsilon}\left(f_{\varepsilon}\right) d y^{\prime} d y \longrightarrow 0
\end{aligned}
$$

as $R \rightarrow+\infty$ uniformly with respect to $\varepsilon \in(0,1)$, thanks to $(2.14),(4.9)$, Lemma 3.1 and Lemma 3.2. Note that a similar argument yields

$$
\left|\iint_{\mathbb{R}_{+}^{2} \backslash[0, R]^{2}}\left(b f^{\prime \prime}-a f f^{\prime}\right)\left(\varphi+\varphi^{\prime}\right) d y d y^{\prime}\right| \longrightarrow 0
$$

as $R \rightarrow \infty$.

Finally, for $R$ large enough, we have

$$
\begin{aligned}
\int_{0}^{\infty} & \int_{0}^{\infty}\left(a_{\varepsilon} f_{\varepsilon} f_{\varepsilon}^{\prime}-b_{\varepsilon} T_{\varepsilon}\left(f_{\varepsilon}\right)\right)\left(T_{\varepsilon}\left(\varphi_{\varepsilon}\right)-\varphi_{\varepsilon}-\varphi_{\varepsilon}^{\prime}\right) d y d y^{\prime} \\
& =\int_{0}^{R} \int_{0}^{R}\left(a_{\varepsilon} f_{\varepsilon} f_{\varepsilon}^{\prime}-b_{\varepsilon} T_{\varepsilon}\left(f_{\varepsilon}\right)\right)\left(T_{\varepsilon}\left(\varphi_{\varepsilon}\right)-\varphi_{\varepsilon}-\varphi_{\varepsilon}^{\prime}\right) d y d y^{\prime} \\
& +\iint_{\mathbb{R}_{+}^{2} \backslash[0, R]^{2}}\left(b_{\varepsilon} T_{\varepsilon}\left(f_{\varepsilon}\right)-a_{\varepsilon} f_{\varepsilon} f_{\varepsilon}^{\prime}\right)\left(\varphi_{\varepsilon}+\varphi_{\varepsilon}^{\prime}\right) d y d y^{\prime}
\end{aligned}
$$

As a conclusion, gathering (4.15), (4.16), (4.17) and (4.18) we are able to pass to the limit in (4.1) and obtain that $f$ satisfies (2.18) ; $f$ is thus a weak solution to the CCF equation (1.1)-(1.2)-(1.3) and the proof of Theorem 2.3 is complete.

\section{A time explicit Euler scheme}

We define an explicit time and size discretisation for the CCF equation (1.1)-(1.3) and prove that the sequence of approximate solutions converges to a solution of the CCF equation (1.1)-(1.3).

Throughout this section we assume that the kinetic coefficients $a, b \in \mathcal{C}\left(\mathbb{R}_{+}^{2}\right)$ satisfy (2.1), (2.2) and (2.3) and fix an initial datum $f^{\text {in }}$ satisfying (2.4). We put

$$
M=\int_{0}^{\infty}(1+y) f^{i n}(y) d y
$$


We will index our approximations by $k \in \mathbb{N} \backslash\{0\}$. For $k \geq 1$ we denote the time discretisation step by $\Delta_{k}$, the size discretisation step by $\varepsilon_{k}$, the number of time steps by $N_{k}$ and the number of size cells by $J_{k} \geq 2$.

For $k \geq 1$ we put

$$
\begin{gathered}
a_{i, j}^{k}=\left\{\begin{array}{ccc}
\varepsilon_{k} a\left(i \varepsilon_{k}, j \varepsilon_{k}\right) & \text { if } & \max \{i, j\} \leq J_{k}, \\
0 & \text { if } & \max \{i, j\}>J_{k},
\end{array}\right. \\
b_{i, j}^{k}=\left\{\begin{array}{ccc}
\varepsilon_{k} b\left(i \varepsilon_{k}, j \varepsilon_{k}\right) & \text { if } & i+j \leq J_{k}, \\
0 & \text { if } & i+j>J_{k} .
\end{array}\right.
\end{gathered}
$$

We define $c^{i n, k}=\left(c_{i}^{i n, k}\right)$ by

$$
c_{i}^{i n, k}=\frac{1}{\varepsilon_{k}} \int_{\Lambda_{i}^{\varepsilon_{k}}} f^{i n}(y) d y, \quad i \in\left\{1, \ldots, 2 J_{k}\right\}
$$

where $\Lambda_{i}^{\varepsilon_{k}}=\left[(i-1 / 2) \varepsilon_{k},(i+1 / 2) \varepsilon_{k}\right), i \geq 1$. If $f^{i n} \in \mathcal{C}\left(\mathbb{R}_{+}\right)$we may also choose $c_{i}^{i n, k}:=f^{i n}\left(i \varepsilon_{k}\right)$ for $i \in\left\{1, \ldots, 2 J_{k}\right\}$. In both cases we have

$$
f^{i n, k}:=\sum_{i=1}^{2 J_{k}} c_{i}^{i n, k} \chi_{i}^{\varepsilon_{k}}(y) \underset{k \rightarrow+\infty}{\longrightarrow} f^{i n} \quad \text { in } \quad L_{1}^{1}\left(\mathbb{R}_{+}\right)
$$

as soon as $\varepsilon_{k} J_{k} \rightarrow+\infty$ as $k \rightarrow+\infty$.

We next consider the following system of $2 J_{k}$ difference equations

$$
\begin{aligned}
\frac{c_{i}^{n+1, k}-c_{i}^{n, k}}{\Delta_{k}} & =Q_{i}^{k}\left(c^{n, k}\right), \quad 0 \leq n \leq N_{k}-1, \\
c_{i}^{0, k} & =c_{i}^{i n, k},
\end{aligned}
$$

for $i \in\left\{1, \ldots, 2 J_{k}\right\}$, where we have set $c^{n, k}=\left(c_{i}^{n, k}\right)_{i \geq 1}$ with $c_{i}^{n, k} \equiv 0$ for $i>2 J_{k}$ and $Q_{i}^{k}($.$) is defined as Q_{i}($.$) with \left(a_{i, j}^{k}\right),\left(b_{i, j}^{k}\right)$ instead of $\left(a_{i, j}\right),\left(b_{i, j}\right)$.

We finally put for each $n \in\left\{0, \ldots, N_{k}-1\right\}$ :

$$
f_{k}(t, y)=\sum_{i=1}^{2 J_{k}} c_{i}^{n, k} \chi_{i}^{\varepsilon_{k}}(y) \quad \text { if } \quad t \in\left[n \Delta_{k},(n+1) \Delta_{k}\right) .
$$

With these notation we may state our convergence result.

Theorem 5.1 There exists $\kappa=\kappa(A, M)$ such that, for any sequences $\left(\Delta_{k}\right),\left(\varepsilon_{k}\right)$, $\left(J_{k}\right),\left(N_{k}\right)$ satisfying

$$
\Delta_{k}, \varepsilon_{k} \rightarrow 0, \quad \varepsilon_{k} J_{k}, \Delta_{k} N_{k} \rightarrow+\infty,
$$




$$
\Delta_{k}\left(\varepsilon_{k} J_{k}\right)^{3}, \Delta_{k} \varepsilon_{k} J_{k} \exp \left(6 A N_{k} \Delta_{k}\right) \leq \kappa,
$$

the sequence $\left(f_{k}\right)$ defined by (5.6) is a sequence of non-negative functions which lies in a weakly compact subset of $L^{1}\left((0, T) \times \mathbb{R}_{+}\right)$and is bounded in $L^{\infty}\left(0, T ; L_{1}^{1}\left(\mathbb{R}_{+}\right)\right)$ for each $T \in \mathbb{R}_{+}$. In addition, up to the extraction of a subsequence, $\left(f_{k}\right)$ converges weakly in $L^{1}\left((0, T) \times \mathbb{R}_{+}\right)$towards a weak solution $f$ to the CCF equation (1.1)-(1.3) for any $T \in \mathbb{R}_{+}$.

A possible choice is

$$
\Delta_{k}=\frac{1}{k}, \quad \varepsilon_{k}=\frac{1}{k}, \quad J_{k}=k^{5 / 4}, \quad N_{k}=\frac{k \ln (k)}{10 \mathrm{~A}} .
$$

Observe that (5.8) strongly couples the admissible choices of the time and size discretisations and implies somehow that keeping a large number of equations (i.e. $J_{k}$ large) requires a very small time step $\Delta_{k}$. This fact was already pointed out in [18] where some instabilities in the numerical simulations are reported when the time step $\Delta_{k}$ is too large with respect to $J_{k}$. Theorem 5.1 thus provides quantitative information on this point. Let us further mention that, in [29], $J_{k}$ is taken to be infinite but the convergence of the scheme is restricted to bounded coagulation coefficients.

The proof of Theorem 5.1 is very similar to the proof of Theorem 2.3. We first establish the non-negativity of the function $f_{k}$ for $k \geq 1$, together with the weak compactness in $L^{1}$ of the sequence $\left(f_{k}\right)$. Without loss of generality we may assume that $\varepsilon_{k} J_{k} \geq 1$ for $k \geq 1$.

Lemma 5.2 The sequence $\left(f_{k}\right)$ satisfies

$$
f_{k}(t, y) \geq 0 \text { for }(t, y) \in \mathbb{R}_{+}^{2}
$$

and, for any $T>0$,

$$
\sup _{[0, T]} \int_{0}^{\infty} f_{k}(t, y)(1+y) d y \leq C_{T} .
$$

Proof. It follows from the symmetry property (2.1), (5.1), (5.2) and (5.4) that

$$
\text { 0) } \sum_{i=1}^{\infty}\left(c_{i}^{n+1, k}-c_{i}^{n, k}\right) \varphi_{i}=\frac{\Delta_{k}}{2} \sum_{i, j=1}^{\infty}\left(a_{i, j}^{k} c_{i}^{n, k} c_{j}^{n, k}-b_{i, j} c_{i+j}^{n, k}\right)\left(\varphi_{i+j}-\varphi_{i}-\varphi_{j}\right)
$$

for any sequence $\left(\varphi_{i}\right)$ of real numbers. Taking $\varphi_{i}=i, i \geq 1$, we clearly get

$$
\varepsilon_{k}^{2} \sum_{i=1}^{\infty} i c_{i}^{n, k}=\varepsilon_{k}^{2} \sum_{i=1}^{\infty} i c_{i}^{i n, k} \leq 2 \int_{0}^{\infty} y f^{i n}(y) d y .
$$

We next claim that

$$
c_{i}^{n, k} \geq 0 \text { for any } n \in\left\{0, \ldots, N_{k}\right\} \text { and } i \geq 1
$$


and

$$
\varepsilon_{k} \sum_{i=1}^{\infty} c_{i}^{n, k} \leq C(A, M) \exp \left\{6 A n \Delta_{k}\right\} .
$$

We proceed by induction. First, (5.12) and (5.13) are obviously true for $n=0$. We next assume that (5.12) and (5.13) hold true for $n^{\prime} \in\{0, \ldots, n\}$ for some $n \in$ $\left\{0, \ldots, N_{k}-1\right\}$. We fix $m_{k} \geq 1$ such that $1 \in \Lambda_{m_{k}}^{\varepsilon_{k}}$ and proceed as in the proof of Lemma 3.2 to obtain that

$$
\sum_{i=1}^{m_{k}+1} \varepsilon_{k}\left(c_{i}^{n^{\prime}+1, k}-c_{i}^{n^{\prime}, k}\right) \leq 6 A \Delta_{k}\left(\sum_{i=1}^{m_{k}+1} \varepsilon_{k} c_{i}^{n^{\prime}, k}+2 M\right)
$$

for any $n^{\prime} \leq n$, where we have used (2.2), (5.11) and the non-negativity of $c_{i}^{n^{\prime}, k}$ for $0 \leq n^{\prime} \leq n$. The discrete Gronwall lemma then yields

$$
\sum_{i=1}^{m_{k}+1} \varepsilon_{k} c_{i}^{n^{\prime}, k} \leq C(A, M) \exp \left\{6 A n^{\prime} \Delta_{k}\right\}
$$

for any $n^{\prime} \leq n+1$, whence (5.13) for $n^{\prime} \in\{0, \ldots, n+1\}$.

On the other hand, either $c_{i}^{n+1, k}=0$ if $i>2 J_{k}$ or $i \leq 2 J_{k}$ and (5.4) and (5.12) for $n$ yield

$$
c_{i}^{n+1, k} \geq\left\{1-\Delta_{k}\left(\frac{1}{2} \sum_{j=1}^{i-1} b_{i-j, j}^{k}+\sum_{j=1}^{\infty} a_{i, j}^{k} c_{j}^{n, k}\right)\right\} c_{i}^{n, k}
$$

with

$$
\frac{1}{2} \sum_{j=1}^{i-1} b_{i-j, j}^{k} \leq A\left(1+2 J_{k} \varepsilon_{k}\right)^{2} J_{k} \varepsilon_{k} \leq 9 A\left(J_{k} \varepsilon_{k}\right)^{3}
$$

by $(2.2)$ and

$$
\begin{aligned}
\sum_{j=1}^{\infty} a_{i, j}^{k} c_{j}^{n, k} & \leq\left(1+2 J_{k} \varepsilon_{k}\right) \sum_{j=1}^{\infty}\left(1+\varepsilon_{k} j\right) \varepsilon_{k} c_{j}^{n, k} \\
& \leq 3 A J_{k} \varepsilon_{k}\left(C(A, M) \exp \left\{6 A n \Delta_{k}\right\}+2 M\right) \\
& \leq C(A, M) J_{k} \varepsilon_{k} \exp \left\{6 A N_{k} \Delta_{k}\right\}
\end{aligned}
$$

by $(2.2),(5.11)$ and (5.13). Consequently,

$$
c_{i}^{n+1, k} \geq\left\{1-C(A, M) \Delta_{k}\left(\left(J_{k} \varepsilon_{k}\right)^{3}+J_{k} \varepsilon_{k} \exp \left\{6 A N_{k} \Delta_{k}\right\}\right)\right\} \geq 0
$$

thanks to (5.8) and the induction argument is complete. We have thus proved that $\left(f_{k}\right)$ satisfies $f_{k} \geq 0$ and

$$
\sup _{[0, T]} \int_{0}^{\infty} f_{k}(t, y)(1+y) d y \leq C_{T}
$$


for any $k \geq 1$.

In order to establish that $\left(f_{k}\right)$ lies in a weakly compact subset of $L^{1}\left((0, T) \times \mathbb{R}_{+}\right)$ we proceed as in Lemma 3.3. We first recall that (2.4) and a refined version of the de la Vallée-Poussin theorem $[9,23]$ ensure that there exists a function $\Phi$ fulfilling the assumptions of Lemma 3.3 and such that $\Phi(u) / u \rightarrow+\infty$ as $u \rightarrow+\infty$ and

$$
\int_{\mathbb{R}_{+}} \Phi\left(f^{i n}\right) d y<+\infty
$$

Lemma 5.3 For every $T \in \mathbb{R}_{+}$and $R \in \mathbb{R}_{+}$, there exists $k_{T}$ such that

$$
\sup _{[0, T]} \int_{0}^{R} \Phi\left(f_{k}(t, y)\right) d y \leq C(T, R) \quad \text { for } k \geq k_{T} .
$$

Proof. We omit the index $k$ for simplicity. Let $m$ be the integer such that $m \varepsilon<$ $R \leq(m+1) \varepsilon$. Since $\Phi$ is convex we infer from (5.4) and the non-negativity of $\left(a_{i, j}\right),\left(b_{i, j}\right)$ and $\left(c_{i}\right)$ that

$$
\begin{aligned}
\sum_{i=1}^{m+1} \varepsilon\left(\Phi\left(c_{i}^{n+1}\right)-\Phi\left(c_{i}^{n}\right)\right) \leq & \varepsilon \sum_{i=1}^{m+1}\left(c_{i}^{n+1}-c_{i}^{n}\right) \Phi^{\prime}\left(c_{i}^{n+1}\right) \\
\leq & \frac{\Delta \varepsilon}{2} \sum_{i=1}^{m+1} \sum_{j=1}^{i-1} a_{j, i-j} c_{i-j}^{n} c_{j}^{n} \Phi^{\prime}\left(c_{i}^{n+1}\right) \\
& +\Delta \varepsilon \sum_{i=1}^{m+1} \sum_{j=1}^{\infty} b_{i, j} c_{i+j}^{n} \Phi^{\prime}\left(c_{i}^{n+1}\right) .
\end{aligned}
$$

On the one hand, thanks to (3.6), (5.9) and the monotonicity of $\Phi$, we have

$$
\begin{aligned}
& \sum_{i=1}^{m+1} \sum_{j=1}^{i-1} a_{j, i-j} c_{i-j}^{n} c_{j}^{n} \Phi^{\prime}\left(c_{i}^{n+1}\right) \\
& \quad \leq A \sum_{i=1}^{m+1} \sum_{j=1}^{i-1} \varepsilon(1+\varepsilon j) c_{j}^{n}(1+\varepsilon(i-j)) c_{i-j}^{n} \Phi^{\prime}\left(c_{i}^{n+1}\right) \\
& \quad \leq A \sum_{i=1}^{m+1} \sum_{j=1}^{i-1} \varepsilon(1+\varepsilon j) c_{j}^{n}\left\{\Phi\left(c_{i}^{n+1}\right)+\Phi\left((1+\varepsilon(i-j)) c_{i-j}^{n}\right)\right\} \\
& \quad \leq C(T)\left\{\sum_{i=1}^{m+1} \Phi\left(c_{i}^{n+1}\right)+\sum_{i=1}^{m+1} \Phi\left((R+2) c_{i}^{n}\right)\right\} .
\end{aligned}
$$

But the concavity of $\Phi^{\prime}$ entails that $\Phi^{\prime}((R+2) u) \leq(R+2) \Phi^{\prime}(u)$ for $u \geq 0$, whence $\Phi((R+2) u) \leq(R+2)^{2} \Phi(u)$. Consequently,

$$
\sum_{i=1}^{m+1} \sum_{j=1}^{i-1} a_{j, i-j} c_{i-j}^{n} c_{j}^{n} \Phi^{\prime}\left(c_{i}^{n+1}\right) \leq C(T) \sum_{i=1}^{m+1} \Phi\left(c_{i}^{n+1}\right)+C(T, R) \sum_{i=1}^{m+1} \Phi\left(c_{i}^{n}\right) .
$$


On the other hand, combining the above argument with the one used in the proof of Lemma 3.3, we obtain

$$
\sum_{i=1}^{m+1} \sum_{j=1}^{\infty} b_{i, j} c_{i+j}^{n} \Phi^{\prime}\left(c_{i}^{n+1}\right) \leq C(T) \sum_{i=1}^{m+1} \Phi\left(c_{i}^{n+1}\right)+\frac{C(T, R)}{\varepsilon} .
$$

Therefore,

$$
\begin{aligned}
\sum_{i=1}^{m+1} \varepsilon \Phi\left(c_{i}^{n+1}\right) \leq \Delta & C(T) \sum_{i=1}^{m+1} \Phi\left(c_{i}^{n}\right)+\Delta C(T, R) \\
& +(1+\Delta C(T, R)) \sum_{i=1}^{m+1} \Phi\left(c_{i}^{n}\right) \\
\sum_{i=1}^{m+1} \varepsilon \Phi\left(c_{i}^{n+1}\right) \leq & \frac{1+\Delta C(T, R)}{1-\Delta C(T)} \sum_{i=1}^{m+1} \Phi\left(c_{i}^{n}\right)+\frac{\Delta C(T, R)}{1-\Delta C(T)} .
\end{aligned}
$$

By (5.7) there is $k_{T}$ such that $\Delta_{k} C_{T} \leq 1 / 2$ for $k \geq k_{T}$. We may then use the discrete Gronwall lemma and argue as in the proof of Lemma 3.3 to conclude that (5.14) holds true.

Finally, the functions $f_{k}$ being discontinuous with respect to time, a weaker version of Lemma 3.5 is available, namely,

$$
\int_{0}^{R} f_{k}(., y) \psi(y) d y \text { is bounded in } B V(0, T)
$$

for any $\psi \in L^{\infty}(0, R), R \in \mathbb{R}_{+}$and $T \in \mathbb{R}_{+}$.

We are now in a position to proceed as in Section 4 to pass to the limit as $k \rightarrow+\infty$ in the equation satisfied by $f_{k}$, which reads, for $\varphi \in \mathcal{D}\left([0,+\infty) \times \mathbb{R}_{+}\right)$and $k$ large enough,

$$
\begin{aligned}
& \int_{\Delta_{k}}^{T} \int_{0}^{\infty} f_{k} \frac{\varphi_{\varepsilon_{k}}-\tau_{\Delta_{k}} \varphi_{\varepsilon_{k}}}{\Delta_{k}} d y d t+\int_{0}^{\Delta_{k}} \int_{0}^{\infty} f^{i n, k} \varphi_{\varepsilon_{k}}(t, .) d y d t \\
& \quad+\frac{1}{2} \int_{0}^{T} \int_{0}^{\infty} \int_{0}^{\infty}\left(a_{k} f_{k} f_{k}^{\prime}-b_{k} T_{\varepsilon_{k}}\left(f_{k}\right)\right)\left(T_{\varepsilon_{k}}\left(\varphi_{\varepsilon_{k}}\right)-\varphi_{\varepsilon_{k}}-\varphi_{\varepsilon_{k}}^{\prime}\right) d y d y^{\prime} d t=0
\end{aligned}
$$

where

$$
a_{k}\left(y, y^{\prime}\right)=\sum_{i, j=1}^{\infty} \frac{a_{i, j}^{k}}{\varepsilon_{k}} \chi_{i}^{\varepsilon_{k}}(y) \chi_{j}^{\varepsilon_{k}}\left(y^{\prime}\right), \quad b_{k}\left(y, y^{\prime}\right)=\sum_{i, j=1}^{\infty} \frac{b_{i, j}^{k}}{\varepsilon_{k}} \chi_{i}^{\varepsilon_{k}}(y) \chi_{j}^{\varepsilon_{k}}\left(y^{\prime}\right),
$$

and $\varphi_{\varepsilon_{k}}(t)$ is the $\varepsilon_{k^{-}}$-step function defined by (1.10), $\tau_{\Delta_{k}} \varphi_{\varepsilon_{k}}(t, y)=\varphi_{\varepsilon_{k}}\left(t-\Delta_{k}, y\right)$ for $\left(t, y, y^{\prime}\right) \in \mathbb{R}_{+}^{3}$. 


\section{Other kinetic coefficients}

In this section we discuss several extensions of Theorem 2.3 under various assumptions on the coagulation and fragmentation rates and first consider the case of sublinear coagulation coefficients as described in Theorem 2.5. In that case, the kinetic coefficients do not necessarily satisfy the growth condition (2.3) which is used to control the behaviour of $f_{\varepsilon}$ for large values of $y$ in the proof of Theorem 2.3. Fortunately it turns out that the assumption (2.22) provides such a control and the following lemma is actually the only new ingredient needed for the approach developed previously to work.

Lemma 6.1 Let $\Phi \in \mathcal{C}^{1}([0,+\infty))$ be a non-negative and piecewise $\mathcal{C}^{2}$-smooth convex function such that $\Phi(0)=0, \Phi^{\prime}(0) \geq 0$ and $\Phi^{\prime}$ is concave. Under the assumptions of Theorem 2.5, if

$$
M_{\Phi}:=\int_{0}^{\infty} \Phi(y) f^{i n}(y) d y<\infty
$$

there holds

$$
\int_{0}^{\infty} \Phi(y) f_{\varepsilon}(t, y) d y \leq C(T), \quad t \in[0, T]
$$

where $C(T)$ depends only on $A, M, \Phi, M_{\Phi}$ and $T$.

Proof. It follows from (6.1) that

$$
\begin{aligned}
\frac{d}{d t} \sum_{i=1}^{\infty} \Phi(i \varepsilon) c_{i}^{\varepsilon} & =\frac{1}{2} \sum_{i, j=1}^{\infty} a_{i, j}^{\varepsilon} c_{i}^{\varepsilon} c_{j}^{\varepsilon}(\Phi((i+j) \varepsilon)-\Phi(i \varepsilon)-\Phi(j \varepsilon)) \\
& -\frac{1}{2} \sum_{i, j=1}^{\infty} b_{i, j}^{\varepsilon} c_{i+j}^{\varepsilon}(\Phi((i+j) \varepsilon)-\Phi(i \varepsilon)-\Phi(j \varepsilon)) .
\end{aligned}
$$

Observe first that the convexity of $\Phi$ and $\Phi(0)=0$ ensure that

$$
\Phi((i+j) \varepsilon) \geq \Phi(i \varepsilon)+\Phi(j \varepsilon), \quad i, j \geq 1,
$$

and recall that the properties enjoyed by $\Phi$ warrant that $\Phi$ satisfies

$$
\left(y+y^{\prime}\right)\left(\Phi\left(y+y^{\prime}\right)-\Phi(y)-\Phi\left(y^{\prime}\right)\right) \leq 2\left(y \Phi\left(y^{\prime}\right)+y^{\prime} \Phi(y)\right)
$$

for $\left(y, y^{\prime}\right) \in \mathbb{R}_{+}^{2}[20$, Lemma A.2]. In addition, (2.22) yields

$$
a_{i, j}^{\varepsilon} \leq A \varepsilon(1+(i+j) \varepsilon), \quad i, j \geq 1
$$

Owing to (6.3), the symmetry of $a_{i, j}^{\varepsilon}$ and the non-negativity of $b_{i, j}^{\varepsilon}$ and $c^{\varepsilon}$ we deduce

$$
\begin{aligned}
\frac{d}{d t} \sum_{i=1}^{\infty} \Phi(i \varepsilon) c_{i}^{\varepsilon} \varepsilon & \leq \frac{1}{2} \sum_{i, j=1}^{\infty} \frac{a_{i, j}^{\varepsilon}}{(i+j)} c_{i}^{\varepsilon} c_{j}^{\varepsilon}(j \varepsilon \Phi(i \varepsilon)+i \varepsilon \Phi(j \varepsilon)) \\
& \leq A\left(\sum_{i=1}^{\infty} \varepsilon(1+i \varepsilon) c_{i}^{\varepsilon}\right)\left(\sum_{i=1}^{\infty} \Phi(i \varepsilon) c_{i}^{\varepsilon} \varepsilon\right)
\end{aligned}
$$


Thanks to (3.1) and (3.2) we finally obtain

$$
\frac{d}{d t} \sum_{i=1}^{\infty} \Phi(i \varepsilon) c_{i}^{\varepsilon} \varepsilon \leq C(T) \sum_{i=1}^{\infty} \Phi(i \varepsilon) c_{i}^{\varepsilon} \varepsilon
$$

whence, by the Gronwall lemma,

$$
\sum_{i=1}^{\infty} \Phi(i \varepsilon) c_{i}^{\varepsilon}(t) \varepsilon \leq C(T) \sum_{i=1}^{\infty} \Phi(i \varepsilon) c_{i}^{i n, \varepsilon} \varepsilon, \quad t \in[0, T] .
$$

Now, the convexity of $\Phi$ and the concavity of $\Phi^{\prime}$ entail that

$$
\Phi(u+v) \leq \Phi(u)+v\left(\Phi^{\prime}(0)+(u+v) \Phi^{\prime \prime}(0)\right), \quad u, v \geq 0,
$$

and thus, since $\Phi$ is increasing, we have

$$
\Phi(y) \leq \Phi\left(i \varepsilon+\frac{\varepsilon}{2}\right) \leq \Phi(i \varepsilon)+C(\Phi)(1+\varepsilon i)
$$

and

$$
\Phi(i \varepsilon) \leq \Phi\left(y+\frac{\varepsilon}{2}\right) \leq \Phi(y)+C(\Phi)(1+\varepsilon i)
$$

for any $y \in \Lambda_{i}^{\varepsilon}$. Consequently,

$$
\begin{aligned}
\int_{0}^{\infty} \Phi(y) f_{\varepsilon}(t, y) d y & \leq \varepsilon \sum_{i=1}^{\infty} c_{i}^{\varepsilon}(\Phi(i \varepsilon)+C(\Phi)(1+\varepsilon i)) \\
& \leq C(T, \Phi) \varepsilon \sum_{i=1}^{\infty} c_{i}^{i n, \varepsilon}(1+\varepsilon i+\Phi(i \varepsilon)) \\
& \leq C(T, \Phi) \int_{0}^{\infty} f^{i n}(y)(1+y+\Phi(y)) d y
\end{aligned}
$$

which completes the proof of Lemma 6.1.

The above computation is formal as the series might not have the required convergence properties. A rigorous justification may be performed by replacing $\Phi$ by $\Phi_{R}$ defined by

$$
\Phi_{R}(y)=\left\{\begin{array}{cl}
\Phi(y) & \text { if } \quad y \in[0, R], \\
\Phi^{\prime}(R)(y-R)+\Phi(R) & \text { if } \quad y \in[R,+\infty),
\end{array}\right.
$$

which enjoys the same properties and then pass to the limit as $R \rightarrow+\infty$.

Proof of Theorem 2.5. Since $y \mapsto y f^{i n}(y)$ belongs to $L^{1}\left(\mathbb{R}_{+}\right)$by $(2.4)$, we use once more a refined version of the de la Vallée-Poussin theorem $[9,23]$ to deduce that there 
exists $\Phi$ satisfying the requirements of Lemma 6.1 and such that $\Phi(u) / u \rightarrow+\infty$ as $u \rightarrow+\infty$ and

$$
\int_{0}^{\infty} \Phi(y) f^{i n}(y) d y<+\infty .
$$

On the one hand, the estimates of Section 3 yields the weak compactness of $\left(f_{\varepsilon}\right)$ in $L^{1}\left((0, T) \times \mathbb{R}_{+}\right)$for each $T \in \mathbb{R}_{+}$. On the other hand, the superlinearity of $\Phi$ at infinity and Lemma 6.1 imply the uniform integrability at infinity of $\left(f_{\varepsilon}\right)$ in $L^{1}\left(\mathbb{R}_{+} ; y d y\right)$. Therefore, $\left(f_{\varepsilon}\right)$ is weakly compact in $L^{1}\left((0, T) \times \mathbb{R}_{+} ;(1+y) d t d y\right)$ for each $T \in \mathbb{R}_{+}$, whence (2.24). This in turn allows us to proceed as in Section 4 to perform the limit $\varepsilon \rightarrow 0$. In addition, since $c^{\varepsilon}$ satisfies (2.23), we have

$$
\int_{0}^{\infty} f_{\varepsilon}(t, y) y d y=\sum_{i=1}^{\infty} \varepsilon^{2} i c_{i}^{\varepsilon}(t)=\sum_{i=1}^{\infty} \varepsilon^{2} i c_{i}^{i n}=\int_{0}^{\infty} f^{i n}(y) \mathcal{I}^{\varepsilon}(y) d y
$$

with $\mathcal{I}^{\varepsilon}(y)=\sum_{i=1}^{\infty} i \chi_{i}^{\varepsilon}(y)$. Since $\left|\mathcal{I}^{\varepsilon}(y)-y\right| \leq \varepsilon / 2 \rightarrow 0$ for any $y \geq 0$ the assertion $(2.25)$ readily follows.

Another situation in which Theorem 2.5 holds is the so-called strong fragmentation case $[7,15]$. More precisely, when the kinetic coefficients satisfy

$$
a\left(y, y^{\prime}\right) \leq A(1+y)^{\alpha}\left(1+y^{\prime}\right)^{\alpha} \quad \text { and } \quad b\left(y, y^{\prime}\right) \geq B\left(1+y+y^{\prime}\right)^{-\beta}
$$

for some $\alpha \in[0,1]$ and $\beta>2(\alpha-1)$, the existence of a solution to the DCF and $\mathrm{CCF}$ equations, satisfying (2.23) and (2.25), respectively, follows from [7] and [15], respectively. Combining the moment estimates in $[7,15]$ and the proof of Theorem 2.3 ensures that Theorem 2.5 holds true in that case, too.

As a final example let us consider the case of multiplicative coagulation coefficients which includes in particular the case $a\left(y, y^{\prime}\right)=y y^{\prime}$. More precisely, assume that $a$ satisfies

$$
r(y) r\left(y^{\prime}\right) \leq a\left(y, y^{\prime}\right) \leq A r(y) r\left(y^{\prime}\right), \quad\left(y, y^{\prime}\right) \in \mathbb{R}_{+}^{2} .
$$

for some non-negative function $r$ and there is a positive function $\beta \in L^{\infty}\left(\mathbb{R}_{+}\right)$such that $\beta(y) \rightarrow 0$ as $y \rightarrow+\infty$ and

$$
b\left(y, y^{\prime}\right) \leq \beta\left(y+y^{\prime}\right), \quad\left(y, y^{\prime}\right) \in \mathbb{R}_{+}^{2} .
$$

In that case, Theorem 2.3 is still valid with the same proof except that one has to control $r f_{\varepsilon}$ for large values of $y$ since $a$ does not necessarily satisfy (2.3). Such a control is supplied by the multiplicative structure (6.8) of $a$ as there holds

$$
\int_{0}^{T}\left(\int_{R+1}^{\infty} r^{\varepsilon}(y) f_{\varepsilon}(t, y) d y\right)^{2} d t \leq C(T)\left(R^{-1}+\sup _{\{y \geq R-1\}}\{\beta(y)\}\right),
$$

for $T \in \mathbb{R}_{+}$and $R \geq 1$ with

$$
r^{\varepsilon}=\sum_{i=1}^{\infty} r_{i}^{\varepsilon} \chi_{i}^{\varepsilon} \text { and } r_{i}^{\varepsilon}=\frac{1}{\varepsilon} \int_{\Lambda_{i}^{\varepsilon}} r(y) d y, \quad i \geq 1 .
$$




\section{Spatially non-homogeneous models}

As already mentioned, the convergence of the family of solutions of the DCF equations to a solution of the CCF equation relies on a weak stability principle. The above convergence result introduced in a spatially homogeneous setting is thus likely to be extended to a spatially non-homogeneous setting in a framework where such a stability result is available. Such a theory has been developed recently in [22] for the diffusive continuous coagulation-fragmentation equation and we present now, without proof, an example of an available convergence result. We refer to [22] for details as well as for other assumptions on the coagulation and fragmentation coefficients (such as a detailed balance condition) for which convergence from the discrete to the continuous coagulation-fragmentation equations could also be obtained. The wellposedness of the diffusive $\mathrm{CCF}$ equation is also investigated in [2] with a different approach.

In the non-homogeneous setting considered here, the clusters are assumed to move in an open bounded subset $\Omega$ of $\mathbb{R}^{N}, N \geq 1$, with smooth boundary $\partial \Omega$, according to brownian movement or diffusion (thermal coagulation). The diffusion coefficient $d=d(y) \in \mathcal{C}\left(\mathbb{R}_{+}\right)>0$ is assumed to be only size-dependent and the diffusive $\mathrm{CCF}$ equation reads

$$
\begin{aligned}
\partial_{t} f-d(y) \Delta_{x} f & =Q(f), \quad(t, x, y) \in(0,+\infty) \times \Omega \times \mathbb{R}_{+}, \\
\partial_{n} f & =0, \quad(t, x, y) \in(0,+\infty) \times \partial \Omega \times \mathbb{R}_{+}, \\
f(0, x, y) & =f^{i n}(x, y), \quad(x, y) \in \Omega \times \mathbb{R}_{+},
\end{aligned}
$$

where $Q(f)$ is still defined by (1.3).

Assume now that, in addition to the symmetry and growth conditions (2.1), (2.2) and (2.3), the following monotonicity condition holds

$$
a\left(y^{\prime}, y-y^{\prime}\right) \leq a\left(y^{\prime}, y\right) \quad \text { for } \quad y \geq y^{\prime} \geq 0
$$

and that the coagulation process dominates the fragmentation process in the following sense

$$
b\left(y^{\prime}, y-y^{\prime}\right) \leq A a\left(y^{\prime}, y\right)+B\left(y^{\prime}\right) \quad \text { for any } \quad y \geq y^{\prime} \geq 0,
$$

where $A$ is a non-negative constant and $B$ is a non-negative function such that

$$
B \in L^{1}\left(\mathbb{R}_{+}\right) \quad \text { and } \quad y \mapsto y B(y) \in L^{\infty}\left(\mathbb{R}_{+}\right) .
$$

We finally consider an initial datum $f^{\text {in }}$ satisfying

$$
f^{i n} \in L^{1}\left(\Omega \times \mathbb{R}_{+} ;(1+y) d x d y\right) \quad \text { is non-negative a.e. }
$$

The above assumptions on $d, a, b$ and $f^{\text {in }}$ then ensure the existence of a weak solution to (7.1)-(7.3) [22, Theorem 2.6]. 
We next introduce a sequence of diffusive DCF equations as follows : for $\varepsilon \in$ $(0,1)$, the kinetic coefficients are still given by $(2.5)$ and we put $d_{i}^{\varepsilon}=d(i \varepsilon)$,

$$
c_{i}^{i n, \varepsilon}(x)=\frac{1}{\varepsilon} \int_{\Lambda_{i}^{\varepsilon}} f^{i n}(x, y) d y, \quad x \in \Omega,
$$

with $\Lambda_{i}^{\varepsilon}=[(i-1 / 2) \varepsilon,(i+1 / 2) \varepsilon)$ for $i \geq 1$. We then denote by $c^{\varepsilon}=\left(c_{i}^{\varepsilon}\right)_{i \geq 1}$ a solution to the diffusive DCF equations

$$
\begin{aligned}
\partial_{t} c_{i}^{\varepsilon}-d_{i}^{\varepsilon} \Delta_{x} c_{i}^{\varepsilon} & =Q_{i}^{\varepsilon}\left(c^{\varepsilon}\right) \text { in }(0,+\infty) \times \Omega, \\
\partial_{n} c_{i}^{\varepsilon} & =0 \text { on }(0,+\infty) \times \partial \Omega, \\
c_{i}^{\varepsilon}(0) & =c_{i}^{i n, \varepsilon} \text { in } \Omega,
\end{aligned}
$$

for $i \geq 1$, where $c_{i}^{\varepsilon}=c_{i}^{\varepsilon}(t, x) \geq 0$ denotes the local concentration of clusters of size $i$. The existence of $c^{\varepsilon}$ follows from, e.g., [21] (see also the references therein for a more precise account of the existence results for the diffusive DCF equations). We finally put

$$
f^{\varepsilon}(t, x, y):=\sum_{i=1}^{\infty} c_{i}^{\varepsilon}(t, x) \chi_{i}^{\varepsilon}(y), \quad(t, x, y) \in \mathbb{R}_{+} \times \Omega \times \mathbb{R}_{+} .
$$

Theorem 7.1 Under the above assumptions, the family $\left(f_{\varepsilon}\right)$ lies in a weakly compact subset of $L^{1}\left((0, T) \times \Omega \times \mathbb{R}_{+}\right)$for each $T \in \mathbb{R}_{+}$and, up to the extraction of a subsequence, $f_{\varepsilon} \rightarrow f$ weakly in $L^{1}\left((0, T) \times \Omega \times \mathbb{R}_{+}\right)$where $f$ is a weak solution to the diffusive CCF (7.1)-(7.3).

\section{A Weak and mild solutions}

Assume that the kinetic coefficients $a$ and $b$ enjoy the symmetry condition (2.1) and the growth condition (2.2) and consider an initial datum satisfying (2.4).

Lemma A.1 For any weak solution $f$ of (1.1)-(1.3) (in the sense of Definition 2.1) there exists $\tilde{f} \in \mathcal{C}^{0,1 / 3}\left([0, T] ; L^{1}\right)$ for any $T \in \mathbb{R}_{+}$such that $\tilde{f}=f$ a.e. in $\mathbb{R}_{+}^{2}$ and $\tilde{f}$ satisfies $\tilde{f}(0)=f^{\text {in }}$ and, for any $t_{1}>t_{0} \geq 0$,

$$
\tilde{f}\left(t_{1}, y\right)-\tilde{f}\left(t_{0}, y\right)=\int_{t_{0}}^{t_{1}} Q(\tilde{f})(s, y) d s \quad \text { for a.e. } y \in \mathbb{R}_{+} .
$$

In other words $\tilde{f}$ is a mild solution to (1.1)-(1.3) in the sense of Definition 2.2.

Proof. We fix $\varepsilon>0$ and a non-negative function $\varrho \in \mathcal{D}\left(\mathbb{R}_{+}\right)$such that $\|\varrho\|_{L^{1}}=1$. We next define

$$
\rho_{\varepsilon}(t)=\frac{1}{\varepsilon} \rho\left(\frac{t}{\varepsilon}\right), \quad f_{\varepsilon}=f *_{t} \rho_{\varepsilon}, \quad Q_{\varepsilon}=Q(f) *_{t} \rho_{\varepsilon} .
$$


It readily follows from $(1.1)$ that $f_{\varepsilon}$ satisfies

$$
\frac{\partial}{\partial t} f_{\varepsilon}=Q_{\varepsilon} \text { in } \mathcal{D}^{\prime}\left([0,+\infty) \times \mathbb{R}_{+}\right) .
$$

Furthermore, the bounds on $f$ and (2.2) imply that, for any $T \in \mathbb{R}_{+}$and $R \in \mathbb{R}_{+}$, we have

$$
Q_{k}(f) \in L^{\infty}\left((0, T) ; L^{1}(0, R)\right) .
$$

We deduce from (A.3) and (A.4) that, for any $\psi \in L^{\infty}\left(\mathbb{R}_{+}\right)$with compact support in $[0,+\infty)$,

$$
\frac{d}{d t} \int_{0}^{\infty} f_{\varepsilon} \psi d y=\int_{0}^{\infty} Q_{\varepsilon} \psi d y \text { in } \mathcal{D}^{\prime}([0,+\infty))
$$

But since

$$
t \mapsto \int_{0}^{\infty} f_{\varepsilon} \psi d y=\left(\int_{0}^{\infty} f \psi d y\right) *_{t} \varrho_{\varepsilon} \in \mathcal{C}([0,+\infty))
$$

we also have for any $t_{1}>t_{0} \geq 0$

$$
\int_{0}^{\infty} f_{\varepsilon}\left(t_{1}\right) \psi d y-\int_{0}^{\infty} f_{\varepsilon}\left(t_{0}\right) \psi d y=\int_{t_{0}}^{t_{1}} \int_{0}^{\infty} Q_{\varepsilon} \psi d y d t .
$$

Consider now $T \geq t_{1}>t_{0} \geq 0$ and $R>0$. On the one hand, we choose $\psi(y)=\operatorname{sign}\left(f_{\varepsilon}\left(t_{1}, y\right)-f_{\varepsilon}\left(t_{0}, y\right)\right) \mathbf{1}_{[0, R]}$ in (A.7) ; this gives

$$
\int_{0}^{R}\left|f_{\varepsilon}\left(t_{1}\right)-f_{\varepsilon}\left(t_{0}\right)\right| d y \leq\left|t_{1}-t_{0}\right|\left\|\varrho_{\varepsilon}\right\|_{L^{1}} \sup _{[0, T]} \int_{0}^{R}|Q(f)| d y
$$

and we notice that $\left\|\varrho_{\varepsilon}\right\|_{L^{1}}=1$ while Definition 2.1 and (2.2) yield

$$
\begin{aligned}
\int_{0}^{R}|Q(f)| d y \leq & \frac{3}{2}\left\{\int_{0}^{R} \int_{0}^{\infty} a\left(y, y^{\prime}\right) f(t, y) f\left(t, y^{\prime}\right) d y d y^{\prime}\right. \\
& \left.\quad+\int_{0}^{\infty} f(t, z) \int_{0}^{\min (z, R)} b(y, z-y) d y d z\right\} \\
\leq & \frac{3 A}{2}\left\{\|f(t)\|_{L_{1}^{1}}\left(\|f(t)\|_{L_{1}^{1}}+(1+R)^{2}\right)\right\} \\
\leq & C(T)(1+R)^{2}
\end{aligned}
$$

for $t \in\left[t_{0}, t_{1}\right]$. Therefore,

$$
\begin{aligned}
\int_{0}^{\infty}\left|f_{\varepsilon}\left(t_{1}\right)-f_{\varepsilon}\left(t_{0}\right)\right| d y & \leq \int_{0}^{R}\left|f_{\varepsilon}\left(t_{1}\right)-f_{\varepsilon}\left(t_{0}\right)\right| d y+\int_{R}^{\infty}\left|f_{\varepsilon}\left(t_{1}\right)-f_{\varepsilon}\left(t_{0}\right)\right| d y \\
& \leq C(T)\left|t_{1}-t_{0}\right|\left(1+R^{2}\right)+\frac{2}{R} \sup _{[0, T]}\|f(t)\|_{L_{1}^{1}}
\end{aligned}
$$

and the choice $R=\left|t_{1}-t_{0}\right|^{-1 / 3}$ leads to

$$
\left\|f_{\varepsilon}\left(t_{1}\right)-f_{\varepsilon}\left(t_{0}\right)\right\|_{L^{1}} \leq C(T)\left|t_{1}-t_{0}\right|^{1 / 3} .
$$


Now the definition of $f_{\varepsilon}$ warrants that $\left(f_{\varepsilon}\right)$ converges towards $f$ in $L^{1}\left((0, T) \times \mathbb{R}_{+}\right)$. Consequently, there is a subsequence of $\left(f_{\varepsilon}\right)$ (not relabeled) such that

$$
f_{\varepsilon}(t, .) \longrightarrow f(t, .) \quad \text { in } L^{1}\left(\mathbb{R}_{+}\right)
$$

for every $t \in[0, T] \backslash \mathcal{Z}$ where $\mathcal{Z}$ is a subset of $[0, T]$ with zero measure. Putting $\tilde{f}(t,)=.f(t,$.$) for t \in[0, T] \backslash \mathcal{Z}$, we infer from (A.8) that

$$
\left\|\tilde{f}\left(t_{1}\right)-\tilde{f}\left(t_{0}\right)\right\|_{L^{1}} \leq C(T)\left|t_{1}-t_{0}\right|^{1 / 3}
$$

for any $t_{0} \in[0, T] \backslash \mathcal{Z}$ and $t_{1} \in[0, T] \backslash \mathcal{Z}$ and we may thus extend $\tilde{f}$ by continuity to a function of $\mathcal{C}^{0,1 / 3}\left([0, T] ; L^{1}\left(\mathbb{R}_{+}\right)\right)$which we still denote by $\tilde{f}$. Clearly $\tilde{f}=f$ a.e. in $\mathbb{R}_{+}^{2}$ and it follows from Definition 2.1 that $\tilde{f}$ also satisfies (2.18). The continuity of $\tilde{f}$ and (2.18) then allow us to conclude that $\tilde{f}(0)=f^{\text {in }}$ and $\tilde{f}$ satisfies (2.20).

Acknowledgments. The second author gratefully acknowledges the partial support of the TMR project Asymptotic Methods in Kinetic Theory ERB FMRX CT97 0157 during this work.

\section{References}

[1] M. Aizenman and T.A. Bak, Convergence to equilibrium in a system of reacting polymers, Comm. Math. Phys. 65 (1979), 203-230.

[2] H. Amann, Coagulation-fragmentation processes, Arch. Rational Mech. Anal. 151 (2000), 339-366.

[3] H. Babovsky, On a Monte Carlo scheme for Smoluchowski's coagulation equation, Monte Carlo Methods Appl. 5 (1999), 1-18.

[4] J.M. Ball and J. Carr, The discrete coagulation-fragmentation equations : existence, uniqueness, and density conservation, J. Statist. Phys. 61 (1990), 203-234.

[5] O. Bruno, A. Friedman and F. Reitich, Asymptotic behavior for a coalescence problem, Trans. Amer. Math. Soc. 338 (1993), 133-158.

[6] I. Bouzoubaa, K. Hamdache and A. Noussair, Global weak solutions via a transportprojection scheme to a thin film growth kinetic model, preprint (1999).

[7] F.P. da Costa, Existence and uniqueness of density conserving solutions to the coagulationfragmentation equations with strong fragmentation, J. Math. Anal. Appl. 192 (1995), 892914 .

[8] M. Deaconu, N. Fournier and E. Tanré, Study of a stochastic particle system associated with the Smoluchowski coagulation equation, preprint, 2001.

[9] C. Dellacherie and P.A. Meyer, Probabilités et Potentiel, Chapitres I à IV, Hermann, Paris, 1975 .

[10] P. Deufhard and M. Wulkow, Computational treatment of polyreaction kinetics by orthogonal polynomials of a discrete variable, Impact Comput. Sci. Engrg. 1 (1989), 269-301.

[11] R.L. Drake, A general mathematical survey of the coagulation equation, in "Topics in Current Aerosol Research (part 2)," International Reviews in Aerosol Physics and Chemistry, Pergamon Press, Oxford, 1972, pp. 203-376. 
[12] P.B. Dubovskiǐ and I.W. Stewart, Existence, uniqueness and mass conservation for the coagulation-fragmentation equation, Math. Methods Appl. Sci. 19 (1996), 571-591.

[13] A. Eibeck and W. Wagner, An efficient stochastic algorithm for studying coagulation dynamics and gelation phenomena, SIAM J. Sci. Comput. 22 (2000), 802-821.

[14] A. Eibeck and W. Wagner, Stochastic particle approximations for Smoluchowski's coagulation equation, preprint, 2000.

[15] M. Escobedo, Ph. Laurençot, S. Mischler and B. Perthame, in preparation.

[16] M. Escobedo, S. Mischler and B. Perthame, Gelation in coagulation and fragmentation models, preprint, 2001.

[17] F. Guiaş, A Monte Carlo approach to the Smoluchowski equations, Monte Carlo Methods Appl. 3 (1997), 313-326.

[18] D.S. Krivitsky, Numerical solution of the Smoluchowski kinetic equation and asymptotics of the distribution function, J. Phys. A 28 (1995), 2025-2039.

[19] Ph. Laurençot, On a class of continuous coagulation-fragmentation equations, J. Differential Equations 167 (2000), 245-274.

[20] Ph. Laurençot, The Lifshitz-Slyozov equation with encounters, Math. Models Methods Appl. Sci. 11 (2001).

[21] Ph. Laurençot and S. Mischler, Global existence for the discrete diffusive coagulationfragmentation equation in $L^{1}$, Rev. Mat. Iberoamericana, to appear.

[22] Ph. Laurençot and S. Mischler, The continuous coagulation-fragmentation equation with diffusion, preprint, 2001.

[23] Lê Châu-Hoàn, Etude de la classe des opérateurs m-accrétifs de $L^{1}(\Omega)$ et accrétifs dans $L^{\infty}(\Omega)$, Thèse de $3^{\text {ème }}$ cycle, Université de Paris VI, 1977.

[24] M.H. Lee, On the validity of the coagulation equation and the nature of runaway growth, Icarus 143 (2000), 74-86.

[25] S. Mischler, Convergence of discrete-velocity schemes for the Boltzmann equation, Arch. Rational Mech. Anal. 140 (1997), 53-77.

[26] H. Müller, Zur allgemeinen Theorie der raschen Koagulation, Kolloidchemische Beihefte 27 (1928), 223-250.

[27] E. Otto and H. Fissan, Brownian coagulation of submicron particles, Advanced Powder Technol. 10 (1999), 1-20.

[28] A. Palczewski and J. Schneider, Existence, stability, and convergence of solutions of discrete velocity models to the Boltzmann equation, J. Statist. Phys. 91 (1998), 307-326.

[29] K.K. Sabelfeld and A.A. Kolodko, Monte Carlo simulation of the coagulation processes governed by Smoluchowski equation with random coefficients, Monte Carlo Methods Appl. 3 (1997), 275-311.

[30] M. Smoluchowski, Drei Vorträge über Diffusion, Brownsche Molekularbewegung und Koagulation von Kolloidteilchen, Physik. Zeitschr. 17 (1916), 557-599.

[31] M. Smoluchowski, Versuch einer mathematischen Theorie der Koagulationskinetik kolloider Lösungen, Zeitschrift f. physik. Chemie 92 (1917), 129-168.

[32] J.L. Spouge, An existence theorem for the discrete coagulation-fragmentation equations, Math. Proc. Cambridge Philos. Soc. 96 (1984), 351-357. 
[33] I.W. Stewart, A global existence theorem for the general coagulation-fragmentation equation with unbounded kernels, Math. Methods Appl. Sci. 11 (1989), 627-648.

[34] I.W. Stewart, A uniqueness theorem for the coagulation-fragmentation equation, Math. Proc. Cambridge Philos. Soc. 107 (1990), 573-578.

[35] I.I. Vrabie, Compactness methods for nonlinear evolutions, Second edition, Pitman Monogr. Surveys Pure Appl. Math. 75, Longman, Harlow, 1995.

[36] R.M. Ziff and E.D. McGrady, The kinetics of cluster fragmentation and depolymerisation, J. Phys. A 18 (1985), 3027-3037. 